\title{
Finding all Nash equilibria of a finite game using polynomial algebra
}

\author{
Ruchira S. Datta
}

Received: 1 December 2006 / Accepted: 28 January 2009 / Published online: 20 February 2009 C) The Author(s) 2009. This article is published with open access at Springerlink.com

\begin{abstract}
The set of Nash equilibria of a finite game is the set of nonnegative solutions to a system of polynomial equations. In this survey article, we describe how to construct certain special games and explain how to find all the complex roots of the corresponding polynomial systems, including all the Nash equilibria. We then explain how to find all the complex roots of the polynomial systems for arbitrary generic games, by polyhedral homotopy continuation starting from the solutions to the specially constructed games. We describe the use of Gröbner bases to solve these polynomial systems and to learn geometric information about how the solution set varies with the payoff functions. Finally, we review the use of the Gambit software package to find all Nash equilibria of a finite game.
\end{abstract}

Keywords Nash equilibrium · Normal form game $\cdot$ Algebraic variety

\section{JEL Classification $\quad$ C72}

Our earlier paper (Datta 2003c) contains much of the material which is surveyed more expansively here. We would like to express our gratitude to the following for generously taking the time to personally discuss with us the use of their software packages: Andrew McLennan and Ted Turocy (Gambit McKelvey et al. 2006), Gert-Martin Greuel (Singular Greuel et al. 2001), and Jan Verschelde (PHC Verschelde 1999). We would also like to thank Gabriela Jeronimo for sending us a preprint of her paper with Daniel Perrucci and Juan Sabia, and Andrew McLennan for suggesting she do so. We would like to thank Richard Fateman and Bernd Sturmfels for supervising the research leading up to that paper, during which the author was partially supported by NSF grant DMS 0138323 . We would also like to acknowledge our debt to Bernd Sturmfels, especially for teaching us about the application of polynomial algebra to Nash equilibria, in the lectures leading to Sturmfels (2002).

\section{R. S. Datta $(\bowtie)$}

QB3 Institute, University of California, 324 Stanley Hall, Berkeley, CA 94720, USA

e-mail: ruchira@berkeley.edu 


\section{Introduction}

The set of Nash equilibria of a finite game is the set of nonnegative solutions to a system of polynomial equations. In this article, we introduce this point of view and survey the computational methods for finding Nash equilibria using polynomial algebra which are available to game theorists today. We give examples which we hope will enable game theorists to find all Nash equilibria of games of relatively larger formats. When we know only a subset of the Nash equilibria of a game, we have a rather shaky foundation for making predictions about what could happen in repeated trials of the game, since play may converge toward a Nash equilibrium which is not in the subset. Knowing all the Nash equilibria of a game will help us to make more principled predictions.

In Sect. 2, we explain the definitions and notation that we use. We prove that the set of all Nash equilibria is given by the nonnegative solutions to a system of polynomial equations, which we describe. In this system some additional variables appear, which we call complementary slack variables, in analogy to linear optimization.

In Sect. 3, we explain what kind of mathematical object the set of Nash equilibria is. After briefly giving definitions of the terms needed, we conclude that it is the quotient of a real algebraic variety by a reflection group. We hope this will suggest to the interested reader some branches of mathematics that may be relevant to game theory. However, this section may be skipped without loss.

In Sect. 4, we eliminate the complementary slack variables from the system. The single system of polynomial equations of Sect. 2 is replaced by a set of systems of polynomial equations, one for each choice of which of the complementary slack variables is nonzero. Each system corresponds to a support of the game. ${ }^{1}$ All Nash equilibria of the game can be found by a combinatorial enumeration of the possible equilibrium supports. Such a support defines, for each pure strategy of a player, whether the respective variable represents a mixed strategy probability, or a slack variable for the suboptimal payoff for that strategy. In the rest of the paper, we explain how to solve a single such system.

In Sect. 5, we note that the polynomial system would be easy to solve if it could be factored. We explain how to construct an instance of the polynomial system which is factorizable. We describe the combinatorial procedure for solving it, carrying it out on examples.

In Sect. 6, we describe the polyhedral subdivision associated with a system of polynomial equations. The reader who has gone through the previous section attentively will be well-equipped to understand this combinatorial notion, which is important for solving polynomial systems generally. The game-theoretic case is a particularly beautiful and clear application because the polynomial system is multilinear, i.e., it has degree at most 1 in each variable. However, this section may be skipped without loss.

In Sect. 7, we explain how to use polyhedral homotopy continuation to find all Nash equilibria of a generic game for which the polynomial system is not necessarily

\footnotetext{
1 Actually the system corresponds to a dual support, i.e., the support of the complementary slack variables. This corresponds to a particular support of the game, along with its boundary.
} 
factorizable, by polyhedral homotopy continuation from one that is. The construction of such a factorizable system was described in Sect. 5. This technique is easily parallelizable. We go through an example using the polyhedral homotopy continuation software PHC.

In Sect. 8, we explain the use of Gröbner basis techniques to find all Nash equilibria of a game parametrically, where the payoffs are the parameters. This gives us geometric insight into how the set of Nash equilibria may vary among games of a particular format. We also mention resultant methods for achieving this objective.

In Sect. 9, we explain the technique used by the game-theoretic software package Gambit to find all Nash equilibria. This technique differs from those previously described. We discuss the advantages and disadvantages of the various techniques.

\section{The system of polynomial equations}

In this section, we will first explain our notation and make the necessary definitions. Then we will describe the system of polynomial equations whose nonnegative solutions are the Nash equilibria.

A monomial in $n$ variables $x_{1}, \ldots, x_{n}$ is an expression of the form $x_{1}^{d_{1}}, \ldots, x_{n}^{d_{n}}$ for some nonnegative integers $d_{1}, \ldots, d_{n}$. It is squarefree if $d_{i} \leq 1$ for all $i$. The degree in $x_{i}$ of this monomial is $d_{i}$, and its total degree is $\sum_{i=1}^{n} d_{i}$. The product of two monomials $x_{1}^{d_{1}} \ldots x_{n}^{d_{n}}$ and $x_{1}^{e_{1}} \ldots x_{n}^{e_{n}}$ is $x_{1}^{d_{1}+e_{1}} \ldots x_{n}^{d_{n}+e_{n}}$.

A polynomial in $n$ variables $x_{1}, \ldots, x_{n}$ with coefficients in a field $K$ is a finite sum of terms. Each term is of the form $\mathrm{cm}$ for some $c \in K$ and some monomial $m$ in $x_{1}, \ldots, x_{n}$. The set of polynomials in $n$ variables is a vector space whose basis is the set of monomials in $n$ variables. Since we can also multiply monomials together, we can extend this multiplication to define a product on the space of polynomials, using commutativity, associativity, and distributivity. This makes the set of polynomials in $n$ variables with coefficients in $K$ into a commutative ring, and the study of such objects is the subject of commutative algebra. A field is a commutative ring in which every nonzero element has a multiplicative inverse.

We can evaluate a polynomial $f=\sum c_{d_{1} \ldots d_{n}} x_{1}^{d_{1}} \ldots x_{n}^{d_{n}}$ at a point $\left(a_{1}, \ldots, a_{n}\right) \in$ $K^{n}$ by substituting $a_{1}, \ldots, a_{n}$ for the variables $x_{1}, \ldots, x_{n}$ respectively, obtaining an expression $\sum c_{d_{1} \ldots d_{n}} a_{1}^{d_{1}} \ldots a_{n}^{d_{n}}$ and carrying out all the multiplications and additions in the field $K$. We denote the resulting element of $K$ as $f\left(a_{1}, \ldots, a_{n}\right)$.

A polynomial equation is an expression $f=g$ for some polynomials $f$ and $g$. A point $a \in K^{n}$ satisfies this equation if $f(a)=g(a)$. Since this is equivalent to $f(a)-g(a)=0$, we can always write a polynomial equation as $p=0$ for some polynomial $p$. A point satisfying this equation is called a root of $p$. A polynomial in one variable with coefficients in $K$ need not have any roots in $K$. But there is always a field containing $K$, called the algebraic closure $\bar{K}$ of $K$, such that every nonconstant univariate polynomial with coefficients in $\bar{K}$ has a root in $\bar{K}$. The field of complex numbers is the algebraic closure of the field of real numbers.

The multiplicity of a root of a polynomial denotes how many linear factors of that polynomial vanish at that root. (Every polynomial factorizes into linear factors over the algebraic closure of its coefficient field.) For example, the multiplicity of the root 
1 of the polynomial $(x-1)^{2}$ is 2 . A polynomial equation of degree $d$ in one variable has $d$ complex roots (counted with multiplicity); this is the Fundamental Theorem of Algebra.

A system of polynomial equations in $n$ variables over $K$ is a finite set of polynomial equations in $n$ variables over $K$, and a point in $K^{n}$ satisfies, or is a root of, this system if it satisfies all the constituent equations. The study of solution sets of polynomial systems is called algebraic geometry. Recent years have seen a renaissance in computational algebraic geometry; the interested reader is referred for example to Cox et al. (1997). The subsequent volume (Cox et al. 198) contains more information, particularly about finding the roots of polynomial systems. The book (Sturmfels 2002) surveys techniques for solving polynomial systems and includes a chapter on finding Nash equilibria. Dickenstein and Emiris (2005) is a recent summary of the state of the art of solving polynomial systems. Sommese and Wampler (2005) explains numerical algebraic geometry and is written for a general technical audience (rather than a mathematical one).

We now fix the game-theoretic notation we shall use. The concepts we describe here can be found in a standard game theory text such as Osborne and Rubinstein (1994). A normal form game with a finite number of players, each with a finite number of pure strategies, is specified as follows. The set of players is denoted as $I=\{1, \ldots, N\}$. Associated to the players are finite disjoint sets of pure strategies $S_{1}, \ldots, S_{N}$. We require that $\left|S_{i}\right| \geq 2$ for each $i$. Write $S=\prod_{i \in I} S_{i}$ for the set of pure strategy profiles. For each $i$ let $d_{i}=\left|S_{i}\right|-1$, and write the set $S_{i}$ as $\left\{s_{i 0}, \ldots, s_{i d_{i}}\right\}$. So a pure strategy profile $s \in S$ can be written as $s=\left(s_{1}, \ldots, s_{N}\right)$, where for each $i$, we have $s_{i}=s_{i j}$ for some $j$ with $0 \leq j \leq d_{i}$. Let $D=\sum_{i \in I}\left(\left|S_{i}\right|-1\right)$. So $\sum_{i \in I}\left|S_{i}\right|=D+N$. The set $\Sigma_{i}$ of mixed strategies of player $i$ is the set of all functions $\sigma_{i}: S_{i} \rightarrow[0,1]$ with $\sum_{j=0}^{d_{i}} \sigma_{i}\left(s_{i j}\right)=1$. Write $\Sigma=\prod_{i \in I} \Sigma_{i}$ for the set of strategy profiles. Write $\Sigma_{-i}=\prod_{j \in I-\{i\}} \Sigma_{j}$. We will call an element of $\Sigma_{-i}$ a $(-i)$-strategy profile. Write $S_{-i}=\prod_{j \in I-\{i\}} S_{j}$. We will call an element of $S_{-i}$ a pure $(-i)$-strategy profile. Write $\sigma_{-i}$ for the image of $\sigma \in \Sigma$ under the projection $\pi_{-i}$ onto $\Sigma_{-i}$. Write $\sigma_{i j}=$ $\sigma_{i}\left(s_{i j}\right)$ for $j=0, \ldots, d_{i}$. A game format is a specification of a number of players $N$ and the number of pure strategies each player has, specified by the numbers $d_{1}, \ldots, d_{N}$. Without loss of generality we can require that $d_{1} \geq d_{2} \geq \cdots \geq d_{N}$.

The game is specified by describing the payoff function $u_{i}: S \rightarrow \mathbb{R}$ for each player. The $i$ th player's expected payoff from a strategy profile $\sigma$ is given by multilinearity as

$$
u_{i}(\sigma)=\sum_{s \in S} u_{i}(s) \sigma_{1}\left(s_{1}\right) \ldots \sigma_{N}\left(s_{N}\right)
$$

By abuse of notation, write $u_{i}\left(\sigma_{i}, \sigma_{-i}\right)$ for the $i$ th player's expected payoff from the strategy $\sigma$ whose $i$ th component is $\sigma_{i}$ and whose other components are defined by $\pi_{-i}(\sigma)=\sigma_{-i}$.

A mixed strategy $\sigma_{i}^{*}$ of player $i$ is a best response to the $(-i)$-strategy profile $\sigma_{-i}$ if for every mixed strategy $\sigma_{i}$ of player $i$, we have $u_{i}\left(\sigma_{i}^{*}, \sigma_{-i}\right) \geq u_{i}\left(\sigma_{i}, \sigma_{-i}\right)$. A strategy profile $\sigma^{*}$ is a Nash equilibrium if for each player $i$, the mixed strategy $\sigma_{i}^{*}$ is a best response to $\sigma_{-i}^{*}$. 
We can rewrite the expected payoff to player $i$ as follows:

$$
\begin{aligned}
u_{i}\left(\sigma_{i}, \sigma_{-i}\right) & =\sum_{s \in S} u_{i}\left(s_{i}, s_{-i}\right) \sigma_{1}\left(s_{1}\right) \cdots \sigma_{N}\left(s_{N}\right) \\
& =\sum_{s_{i} \in S_{i}} \sum_{s_{-i} \in S_{-i}} u_{i}\left(s_{i}, s_{-i}\right) \sigma_{1}\left(s_{1}\right) \cdots \sigma_{N}\left(s_{N}\right) \\
& =\sum_{s_{i} \in S_{i}} \sigma_{i}\left(s_{i}\right) \sum_{s_{-i} \in S_{-i}} u_{i}\left(s_{i}, s_{-i}\right) \sigma_{1}\left(s_{1}\right) \cdots \sigma_{i-1}\left(s_{i-1}\right) \sigma_{i+1}\left(s_{i+1}\right) \cdots \sigma_{N}\left(s_{N}\right) \\
& =\sum_{j=0}^{d_{i}} \sigma_{i j} u_{i}\left(s_{i j}, \sigma_{-i}\right) .
\end{aligned}
$$

We see that the function $u_{i}\left(s_{i j}, \sigma_{-i}\right)$ is a polynomial with real-valued coefficients. For each $s_{-i}=\left(s_{1 j_{1}}, \ldots, s_{(i-1) j_{i-1}}, s_{(i+1) j_{i+1}}, \ldots, s_{N j_{N}}\right) \in S_{-i}$, the contribution to $u_{i}\left(s_{i j}, \sigma_{-i}\right)$ from the outcome $\left(s_{i j}, s_{-i}\right)$ is the squarefree monomial

$$
\sigma_{1 j_{1}} \cdots \sigma_{(i-1) j_{i-1}} \sigma_{(i+1) j_{i+1}} \cdots \sigma_{N j_{N}}
$$

(which may be interpreted as the conditional probability that the outcome will occur, given that player $i$ chooses pure strategy $s_{i j}$ ), multiplied by the real-valued coefficient $u_{i}\left(s_{i j}, s_{-i}\right)$ (the payoff to player $i$ for that outcome). The following proposition describes the system of polynomial equations whose nonnegative solutions give the Nash equilibria.

Proposition 1 The sequence of real numbers

$$
\left(\sigma_{10}, \ldots, \sigma_{1 d_{1}}, \ldots, \sigma_{N 0}, \ldots, \sigma_{N d_{N}}\right)
$$

constitutes a Nash equilibrium if and only if for some sequence of real numbers

$$
\left(v_{10}, \ldots, v_{1 d_{1}}, \ldots, v_{N 0}, \ldots, v_{N d_{N}}\right)
$$

the $\sigma_{i j}$ 's and $v_{i j}$ 's satisfy the following system $(*)$ of $2(D+N)$ polynomial equations in $2(D+N)$ unknowns:

$$
\begin{array}{rlrl}
u_{i}\left(s_{i j}, \sigma_{-i}\right)+v_{i j} & =u_{i}\left(s_{i 0}, \sigma_{-i}\right)+v_{i 0} \quad \text { for each } i \in I \text { and for } j=1, \ldots, d_{i}, \\
\sigma_{i j} v_{i j} & =0 & \text { for each } i \in I \text { and for } j=0, \ldots, d_{i}, \\
\sum_{j=0}^{d_{i}} \sigma_{i j} & =1 & \text { for each } i \in I,
\end{array}
$$

and all the $\sigma_{i j}$ 's and $v_{i j}$ 's are nonnegative.

Proof $\Longrightarrow$ : Suppose $\sigma$ is a Nash equilibrium. Certainly all the $\sigma_{i j}$ 's are nonnegative and $\sum_{j=0}^{d_{i}} \sigma_{i j}=1$ for each $i \in I$. For each $i \in I$ and $j=0, \ldots, d_{i}$, let 
$v_{i j}=u_{i}(\sigma)-u_{i}\left(s_{i j}, \sigma_{-i}\right)$. Then $v_{i j} \geq 0$ since $\sigma_{i}$ is a best response to $\sigma_{-i}$. The quantities $u_{i}\left(s_{i j}, \sigma_{-i}\right)+v_{i j}$ are all equal to $u_{i}(\sigma)$ and hence to each other.

It remains to show that $\sigma_{i j} v_{i j}=0$.

$$
\begin{aligned}
\sigma_{i j} v_{i j} & =\sigma_{i j}\left(u_{i}(\sigma)-u_{i}\left(s_{i j}, \sigma_{-i}\right)\right) \\
& =\sigma_{i j}\left(u_{i}(\sigma)-u_{i}\left(s_{i j}, \sigma_{-i}\right)\right)-u_{i}(\sigma)+\sum_{l=0}^{d_{i}} \sigma_{i l} u_{i}\left(s_{i l}, \sigma_{-i}\right) \\
& =\left(\sum_{\substack{l=0 \\
l \neq j}}^{d_{i}} \sigma_{i l} u_{i}\left(s_{i l}, \sigma_{-i}\right)\right)-\left(1-\sigma_{i j}\right) u_{i}(\sigma) \\
& =\left(\sum_{\substack{l=0 \\
l \neq j}}^{d_{i}} \sigma_{i l} u_{i}\left(s_{i l}, \sigma_{-i}\right)\right)-\left(\sum_{\substack{l=0 \\
l \neq j}}^{d_{i}} \sigma_{i l}\right) u_{i}(\sigma) \\
& =\sum_{\substack{l=0 \\
l \neq j}}^{d_{i}} \sigma_{i l}\left(u_{i}\left(s_{i l}, \sigma_{-i}\right)-u_{i}(\sigma)\right) .
\end{aligned}
$$

Since $\sigma$ is a best response to $\left(s_{i l}, \sigma_{-i}\right)$ for each $l$, we have $u_{i}\left(s_{i l}, \sigma_{-i}\right)-u_{i}(\sigma) \leq 0$ for each $l$. Since the $\sigma_{i j}$ 's and $v_{i j}$ 's are nonnegative, we must have $\sigma_{i j} v_{i j}=0$.

$\Longleftarrow$ : Suppose the $\sigma_{i j}$ 's and $v_{i j}$ 's are nonnegative and satisfy the polynomial system. Let $\sigma$ be the strategy profile defined by $\sigma_{i}\left(s_{i j}\right)=\sigma_{i j}$. Fix a player $i$ and suppose $\sigma_{i}^{\prime}$ is a mixed strategy of player $i$. Then $\sum_{j=0}^{d_{i}}\left(\sigma_{i j}^{\prime}-\sigma_{i j}\right)=0$, so there must be some $j$ for which $\sigma_{i j}^{\prime}-\sigma_{i j} \geq 0$. Without loss of generality suppose that $\sigma_{i 0}^{\prime}-\sigma_{i 0} \geq 0$. Then

$$
\begin{aligned}
u_{i}(\sigma)-u_{i}\left(\sigma_{i}^{\prime}, \sigma_{-i}\right) & =\sum_{j=0}^{d_{i}}\left(\sigma_{i j}-\sigma_{i j}^{\prime}\right) u_{i}\left(s_{i j}, \sigma_{-i}\right) \\
& =\sum_{j=0}^{d_{i}}\left(\sigma_{i j}-\sigma_{i j}^{\prime}\right)\left(u_{i}\left(s_{i j}, \sigma_{-i}\right)-u_{i}\left(s_{i 0}, \sigma_{-i}\right)\right) \\
& =\sum_{j=1}^{d_{i}}\left(\sigma_{i j}-\sigma_{i j}^{\prime}\right)\left(v_{i 0}-v_{i j}\right) \\
& =\left(\left(1-\sigma_{i 0}\right)-\left(1-\sigma_{i 0}^{\prime}\right)\right) v_{i 0}+\sum_{j=1}^{d_{i}} \sigma_{i j}^{\prime} v_{i j} \\
& =\left(\sigma_{i 0}^{\prime}-\sigma_{i 0}\right) v_{i 0}+\sum_{j=1}^{d_{i}} \sigma_{i j}^{\prime} v_{i j} \geq 0 .
\end{aligned}
$$

Thus $\sigma_{i}$ is a best response to $\sigma_{-i}$ for each $i$, and $\sigma$ is a Nash equilibrium. 
In the statement of the proposition, we have included $D$ polynomial equations $u_{i}\left(s_{i j}, \sigma_{-i}\right)+v_{i j}=u_{i}\left(s_{i 0}, \sigma_{-i}\right)+v_{i 0}$, singling out the 0th pure strategy of the $i$ th player. In fact, these $D$ polynomial equations imply the polynomial equations $u_{i}\left(s_{i j}, \sigma_{-i}\right)+v_{i j}=u_{i}\left(s_{i l}, \sigma_{-i}\right)+v_{i l}$ for all pairs of pure strategies $s_{i j}$ and $s_{i l}$ of the $i$ th player. We have picked these $D$ polynomial equations since they are sufficient, and to emphasize that we have the same number of equations as unknowns.

We call the $v_{i j}$ 's complementary slack variables. If $v_{i j}>0$, that is, the payoff $u_{i}\left(s_{i j}, \sigma_{-i}\right)$ to player $i$ for strategy $s_{i j}$ is strictly less than the equilibrium payoff $u_{i}(\sigma)$, then $\sigma_{i j}=0$, that is, strategy $s_{i j}$ cannot be a component of the mixed strategy of player $i$. Conversely, if $\sigma_{i j}>0$ (which must hold for some $j$ ), then the payoff to player $i$ of pure strategy $s_{i j}$ must equal the equilibrium payoff.

\section{What kind of geometric object is the set of Nash equilibria?}

In this section, we explain what kind of geometric object the set of Nash equilibria is, briefly explaining the needed mathematical concepts as we go. We hope that this will suggest to the interested reader some of the branches of mathematics that may be useful for the game theorist, but the reader may skip this section without loss, referring back to it only for unfamiliar terms.

We have seen that the set of Nash equilibria is the set of real solutions to a system of polynomial equations and inequalities with real coefficients. Inequalities can only be defined over ordered sets; the field of complex numbers, for example, is not ordered. A set of real points given by a system of polynomial equations and inequalities is called a semialgebraic variety, and the special case when the system does not involve inequalities is called a real algebraic variety. Thus the set of Nash equilibria of a game is a semialgebraic variety. Real algebraic geometry is the study of real algebraic varieties and semialgebraic varieties. This area of algebraic geometry has many features of special interest (quite apart from its usefulness in applications). (For example, over the real numbers any system of equations $f_{1}=0, \ldots, f_{n}=0$ is equivalent to a single equation $f_{1}^{2}+f_{2}^{2}+\cdots+f_{n}^{2}=0$.) It so happens that Nash's contribution to real algebraic geometry was seminal, although he did not relate it to game theory. The two main references for real algebraic geometry are Bochnak et al. (1998) and Basu et al. (2003).

The set of points in the plane with $x \geq 0$ and $y \geq 0$ is the nonnegative quadrant, the set of points in 3-space with $x \geq 0, y \geq 0$, and $z \geq 0$ is the nonnegative octant, and similarly the set of points in $\mathbb{R}^{n}$ all of whose coordinates are nonnegative is the nonnegative orthant. In this case, the inequalities state simply that we are interested in those solutions to the polynomial system which lie in the nonnegative orthant.

In the system $(*)$ of polynomial equations, we can substitute each $\sigma_{i j}$ with $\rho_{i j}^{2}$ and each $v_{i j}$ with $r_{i j}^{2}$, where the $\rho_{i j}$ 's and the $r_{i j}$ 's are new unknowns. This induces a new system $(* *)$ of polynomial equations in the $\rho_{i j}$ 's and $r_{i j}$ 's. (For example, $\rho_{i j}^{2} r_{i j}^{2}=0$, $\sum_{j=0}^{d_{i}} \rho_{i j}^{2}=1$, and so forth.) Each real-valued solution to $(* *)$ corresponds to a Nash equilibrium, since $\sigma_{i j}=\rho_{i j}^{2}$ and $v_{i j}=r_{i j}^{2}$ automatically satisfy the nonnegativity constraints. However, there may be up to $2^{D+N}$ solutions to (**) for each Nash 
equilibrium, since if $\sigma_{i j}$ is positive we can set each $\rho_{i j}$ equal to either its positive or negative square root, and similarly for $v_{i j}$.

A transformation of a set $X$ is a 1-1 correspondence of $X$ with itself. A group of transformations is a set $G$ of transformations such that for each transformation in $G$, its inverse transformation is also in $G$, and for each pair of transformations in $G$, their composition is also in $G$. We consider the composition of two transformations in $G$ to be their product in $G$. (Note that this product is not necessarily commutative.) We say that the group of transformations acts on the set $X$ which it transforms. Given any set $T$ of transformations $\left\{g_{1}, \ldots, g_{n}\right\}$, we can form the group generated by the generators $g_{1}, \ldots, g_{n}$ by taking all products of elements of $T$ and their inverses. An orbit of the group action is the set of images of a single point in $X$ under all the transformations in $G$. Every point in an orbit is the image of every other point under some transformation in $G$. To form the quotient of $X$ by $G$, we can take one point from each orbit.

Each $(n-1)$-dimensional hyperplane in $\mathbb{R}^{n}$ defines a special transformation, the reflection which takes each point in $\mathbb{R}^{n}$ to its opposite point on the other side of the hyperplane. In particular, the transformation of $\mathbb{R}^{2(D+N)}$ which takes $\rho_{i j}$ into $-\rho_{i j}$ and leaves all other coordinates unchanged is a coordinate reflection, as is the one which takes $r_{i j}$ into $-r_{i j}$. A group of transformations generated by reflections is called a reflection group. In particular, let $G$ be the group of transformations generated by the aforementioned coordinate reflections. Then for any transformation $g \in G$, for any real-valued solution $(\rho, r)$ of $(* *)$, its image $g(\rho, r)$ also satisfies $(* *)$. Indeed, $g$ simply changes the signs of some of the coordinates of $(\rho, r)$. Thus the set of realvalued solutions to (**) is a symmetric real algebraic variety $V$, with $G$ as its group of symmetries.

We can take the quotient of $V$ by $G$ by, for example, considering only those points of $V$ lying in the nonnegative orthant. The group $G$ takes this orthant to each other orthant. There is exactly one point of $V$ in the nonnegative orthant for each Nash equilibrium, obtained by taking the nonnegative square root of each coordinate of the Nash equilibrium. So the set of Nash equilibria is the quotient of a real algebraic variety by a reflection group. In fact, any semialgebraic variety defined by a system of polynomial equations and inequalities in which none of the inequalities are strict, is also the quotient of a real algebraic variety by a reflection group. For each inequality constraint $f\left(x_{1}, \ldots, x_{n}\right) \geq 0$, we can define a new variable $v$ and change the inequality constraint into two constraints $f\left(x_{1}, \ldots, x_{n}\right)-v=0$ and $v \geq 0$, then proceed as above.

\section{Eliminating the complementary slack variables}

In this section, we will eliminate the complementary slack variables from the system $(*)$. We arrive at a set of polynomial systems $(E)$. Those solutions of one of the systems $(E)$ which satisfy all the nonnegativity constraints (including the ones on the complementary slack variables) are Nash equilibria. In the rest of the paper, we will explain how to solve such a polynomial system $(E)$.

Elimination theory in commutative algebra solves polynomial systems by eliminating variables using Gröbner basis techniques, as we describe later on. But in this case we can eliminate the complementary slack variables using only elementary methods. 
In the appendix to this paper, we carry out steps similar to those carried out by a Gröbner basis algorithm to eliminate the complementary slack variables, again using only elementary methods. The interested reader may wish to peruse the appendix to get a flavor of what Gröbner basis algorithms do. The appendix concludes that for a generic game, at each solution either for every player all but one of the complementary slack variables are positive, corresponding to a pure strict Nash equilibrium, or for at least two players at least two of them are zero. (The meaning of "generic" used here is also defined.) Finding pure strict Nash equilibria is a combinatorial procedure which does not require any polynomial algebra. Therefore we do not discuss it further in this article, but assume that we have already found all pure strict Nash equilibria (if any exist), and are now interested in finding the other Nash equilibria, which requires solving polynomial systems.

Any solution to the system $(*)$ induces a partition of $P \stackrel{\text { def }}{=} \cup_{i \in I} S_{i}$ into two subsets, the subset $P_{0}$ such that $v_{i j}=0$ for all $s_{i j} \in P_{0}$ and the subset $P_{+}$such that $v_{i j}>0$ for all $s_{i j} \in P_{+}$. If we make a choice of such a partition, then $\sigma_{i j}=0$ for all $s_{i j} \in P_{+}$, so eliminating these strategies and considering the reduced game, the system $(*)$ reduces to the system $(\mathbf{E})$ :

$$
\begin{aligned}
& u_{i}\left(s_{i j}, \sigma_{-i}\right)=u_{i}\left(s_{i 0}, \sigma_{-i}\right) \quad \text { for each } i \in I \text { and for } j=1, \ldots, d_{i}, \quad\left(\mathbf{E}_{i j}\right) \\
& \sum_{j=0}^{d_{i}} \sigma_{i j}=1 \quad \text { for each } i \in I,
\end{aligned}
$$

and all the $\sigma_{i j}$ 's are nonnegative. (A root of the polynomial equations of the system (E) which does not satisfy the nonnegativity constraints, perhaps because it is not even real, is called a quasi-equilibrium.) After solving the system (E) to find a candidate $\sigma$, we have to check that for each strategy $s_{i j}$ of the original game which we had eliminated, $v_{i j}=u_{i}(\sigma)-u_{i}\left(s_{i j}, \sigma_{-i}\right)$ is indeed nonnegative. In that case $\sigma$ is a solution to the original system $(*)$ and hence a Nash equilibrium of the original game. To find all the Nash equilibria, we can perform this procedure for all partitions $\left(P_{0}, P_{+}\right)$for which at least one $s_{n m} \in P_{0}$ for each player $n$. As noted above, for a generic game there will not be a Nash equilibrium for which there is exactly one player for which at least two $s_{i j}$ 's are in $P_{0}$.

From now on we will restrict our attention to solving systems of the form $(\mathbf{E})$. The paper (McKelvey and McLennan 1997) describes this system, which along with the constraints $\sigma_{i j}>0$ for all the $\sigma_{i j}$ 's, gives the totally mixed Nash equilibria. The Gambit software package (McKelvey et al. 2006) finds all Nash equilibria recursively, by finding totally mixed Nash equilibria of each reduced game by solving the corresponding system. This algorithm for finding all Nash equilibria is described for example in Herings and Peeters (2005) and Datta (2003a).

\section{Solving an instance of the polynomial system}

In this section, we begin considering how to solve systems of the form $(\mathbf{E})$, by looking at certain special systems which are relatively easy to solve. We shall see later that this is the first step in solving generic systems. 
For any $s \in S$, with $s_{i}=s_{i j_{i}}$ for each $i \in I$, we write

$$
\begin{aligned}
u_{j_{1} \ldots j_{N}}^{i}= & u_{i}\left(s_{1 j_{1}}, s_{2 j_{2}}, \ldots, s_{(i-1) j_{i-1}}, s_{i j_{i}}, s_{(i+1) j_{i+1}}, \ldots, s_{N j_{N}}\right) \\
& -u_{i}\left(s_{1 j_{1}}, s_{2 j_{2}}, \ldots, s_{(i-1) j_{i-1}}, s_{i 0}, s_{(i+1) j_{i+1}}, \ldots, s_{N j_{N}}\right) .
\end{aligned}
$$

In particular $u_{j_{1} \ldots j_{N}}^{i}=0$ if $j_{i}=0$. Then the equation $\left(\mathbf{E}_{i j}\right)$ is

$$
\sum_{j_{1}=0}^{d_{1}} \ldots \sum_{j_{i-1}=0}^{d_{i-1}} \sum_{j_{i+1}=0}^{d_{i+1}} \ldots \sum_{j_{N}=0}^{d_{N}} u_{j_{1} \ldots j_{i-1} j j_{i+1} \ldots j_{N}}^{i} \sigma_{1 j_{1}} \ldots \sigma_{(i-1) j_{i-1}} \sigma_{(i+1) j_{i+1}} \ldots \sigma_{N j_{N}}=0
$$

How do we solve an equation like this? We note in passing that if all the coefficients $u_{j_{1} \ldots j_{i-1} j j_{i+1} \ldots j_{N}}^{i}$ had the same sign, we would know that either all the monomials vanished, or $\sigma_{i j}=0$ and $v_{i j}>0$. However, this condition depends on the choice of which strategy in $S_{i}$ to label as $s_{i 0}$, so to check whether it ever arises would require checking the difference between every pair $u_{i}\left(s_{i} j_{1}, s_{-i}\right)-u_{i}\left(s_{i} j_{0}, s_{-i}\right)$.

Well, if the equation factored, it would be easy to solve. That is, if we could find numbers $\mu_{k j_{k}}^{(i j)}$ for $k \in I-\{i\}$ and $s_{k j_{k}} \in S_{k}$ such that

$$
\begin{aligned}
& \sum_{j_{1}=0}^{d_{1}} \ldots \sum_{j_{i-1}=0}^{d_{i-1}} \sum_{j_{i+1}=0}^{d_{i+1}} \ldots \sum_{j_{N}=0}^{d_{N}} u_{j_{1} \ldots j_{i-1} j j_{i+1} \ldots j_{N}}^{i} \sigma_{1 j_{1}} \ldots \sigma_{(i-1) j_{i-1}} \sigma_{(i+1) j_{i+1}} \ldots \sigma_{N j_{N}} \\
& \quad=\prod_{k \in I-\{i\}}\left(\sum_{j_{k}=0}^{d_{k}} \mu_{k j_{k}}^{(i j)} \sigma_{k j_{k}}\right)
\end{aligned}
$$

then we could solve the equation by setting $\sum_{j_{k}=0}^{d_{k}} \mu_{k j_{k}}^{(i j)} \sigma_{k j_{k}}$ equal to zero for some $k \in I-\{i\}$.

The factorization holds if and only if the coefficients of each monomial

$$
\sigma_{1 j_{1}} \ldots \sigma_{(i-1) j_{i-1}} \sigma_{(i+1) j_{i+1}} \ldots \sigma_{N j_{N}}
$$

on both sides of the equation are equal. The coefficient on the left-hand side is $u_{j_{1} \ldots j_{i-1} j j_{i+1} \ldots j_{N}}^{i}$, and the coefficient on the right-hand side is $\prod_{k \in I-\{i\}} \mu_{k j_{k}}^{(i j)}$. Thus we have a system of equations $\prod_{k \in I-\{i\}} \mu_{k j_{k}}^{(i j)}=u_{j_{1} \ldots j_{i-1} j j_{i+1} \ldots j_{N}}^{i}$. Equivalently we have the system of linear equations $\sum_{k \in I-\{i\}} \log \left|\mu_{k j_{k}}^{(i j)}\right|=\log \left|u_{j_{1} \ldots j_{i-1} j j_{i+1} \ldots j_{N}}^{i}\right|$ together with sign conditions $\prod_{k \in I-\{i\}} \operatorname{sign}\left(\mu_{k j_{k}}^{(i j)}\right)=\operatorname{sign}\left(u_{j_{1} \ldots j_{i-1} j j_{i+1} \ldots j_{N}}^{i}\right)$. Unfortunately this linear system is overdetermined and hence usually inconsistent. We have $\prod_{\substack{k=1 \\ k \neq i}}^{N}\left(d_{i}+1\right)$ equations in only $\sum_{\substack{k=1 \\ k \neq i}}^{N}\left(d_{i}+1\right)$ unknowns $\mu_{k j_{k}}^{(i j)}$. So in general, equation $\left(\mathbf{E}_{i j}\right)$ does not factorize.

Nevertheless, suppose all the equations $\left(\mathbf{E}_{i j}\right)$ did factorize. How would we solve the whole system $(\mathbf{E})$ then? For one thing we would substitute $\sigma_{k 0}=1-\sum_{j_{k}=1}^{d_{k}} \sigma_{k j_{k}}$ 
into each linear factor $\sum_{j_{k}=0}^{d_{k}} \mu_{k j_{k}}^{(i j)} \sigma_{k j_{k}}$ to get an affine linear factor

$$
\mu_{k 0}^{(i j)}+\sum_{j=1}^{d_{k}}\left(\mu_{k j_{k}}^{(i j)}-\mu_{k 0}^{(i j)}\right) \sigma_{k j_{k}}
$$

("Affine" just means that it includes a constant term.) We set $\lambda_{k j_{k}}^{(i j)}=\mu_{k j_{k}}^{(i j)}-\mu_{k 0}^{(i j)}$ for $j_{k}=1, \ldots, d_{k}$ and $\lambda_{k 0}^{(i j)}=\mu_{k 0}^{(i j)}$.

We will now construct a particular system in which all the equations factorize, and solve that. In this case the solution method reduces to a combinatorial choice of which set of linear factors to set to zero. For this purpose, it will be convenient to have available a totally nonsingular matrix. An $m \times n$ matrix $M=\left(m_{i j}\right)$ is totally nonsingular if for every $k \leq \min (m, n)$, for every subset $R \subseteq\{1, \ldots, m\}$ with $|R|=k$ and every subset $C \subseteq\{1, \ldots, n\}$ with $|C|=k$, the $k \times k$ submatrix of $M$ given by $\left(m_{i j}\right)_{\substack{i \in R \\ j \in C}}$ is nonsingular.

Let $f: \mathbb{N} \rightarrow \mathbb{R}_{>0}$ be any injection of $\mathbb{N}$ into $\mathbb{R}_{>0}$. We can use the algorithm in Fig. 1 to construct a totally singular $n \times n$ matrix for any $n$. We start with the $1 \times 1$ matrix $\left(m_{11}\right)=(f(1))$, which is clearly totally nonsingular. The problem of filling in a matrix while maintaining some condition is called a matrix completion problem. Torregrosa et al. (2007) shows that we can construct a totally nonsingular matrix by filling in the entries one at a time, as in the algorithm in Fig. 1. In fact, at each stage there are only finitely many possible values of the next entry which would violate the condition, so all we have to do is avoid those values. Therefore each while loop in the algorithm will always terminate. Since the partially filled-in matrix is symmetric, and $m_{i j}$ does not violate the condition, setting $m_{j i}=m_{i j}$ cannot violate it either, and we can keep the matrix symmetric. For example, here is the totally nonsingular $6 \times 6$ matrix given by the algorithm in Fig. 1 , with $f(k)=2^{k-1}$ :

$$
\left[\begin{array}{rrrrrr}
1 & 2 & 4 & 8 & 16 & 32 \\
2 & -4 & 16 & -32 & 128 & -256 \\
4 & 16 & -16 & -128 & 1024 & -256 \\
8 & -32 & -128 & -64 & 4096 & 4096 \\
16 & 128 & 1024 & 4096 & -256 & 1024 \\
32 & -256 & -256 & 4096 & 1024 & -1024
\end{array}\right]
$$

As a matter of fact, a random matrix will be totally nonsingular with probability one. However, if we do use a random matrix we should check that it is indeed totally nonsingular. Since checking this may take a long time, it may be useful to build a large totally nonsingular matrix once and for all and keep it around.

Now assume we have a totally nonsingular $D \times D$ matrix $M$ with entries $m_{i j}$. Define $n(i, j)=j+\sum_{k=1}^{i-1} d_{k}$. So if we write the equations $\left(\mathbf{E}_{i j}\right)$ in sequence $\left(\mathbf{E}_{11}\right), \ldots$, $\left(\mathbf{E}_{1 d_{1}}\right), \ldots,\left(\mathbf{E}_{N 1}\right), \ldots,\left(\mathbf{E}_{N d_{N}}\right)$, then the $n(i, j)$ th equation in the sequence is $\left(\mathbf{E}_{i, j}\right)$. Set $\lambda_{k l_{k}}^{(i j)}=m_{n(i, j) l_{k}}$ for $l_{k}>0$ and $\lambda_{k 0}^{(i j)}=-1$. This defines a particular system $(\mathbf{S})$ 


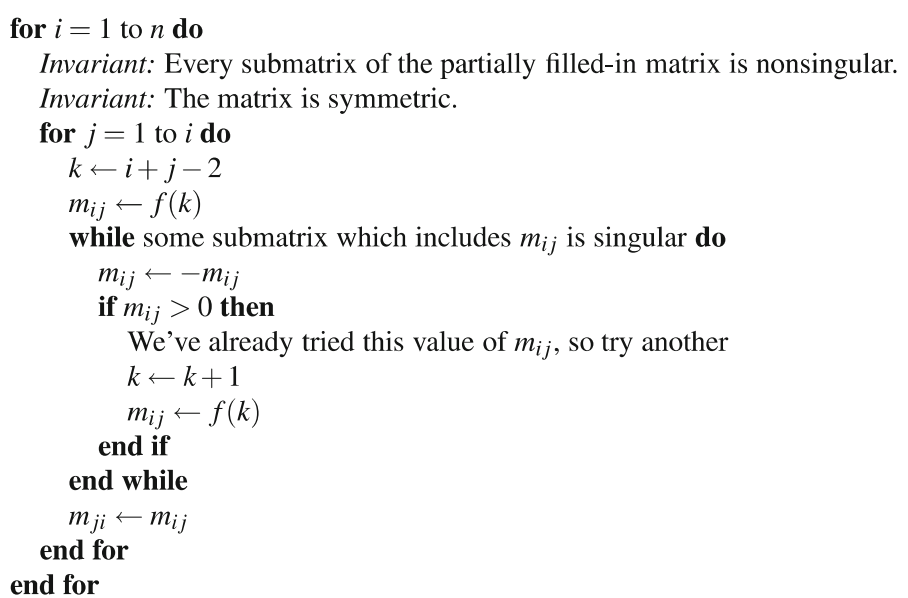

Fig. 1 One possible algorithm to compute a totally nonsingular matrix

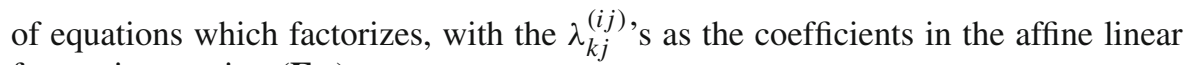
factors in equation $\left(\mathbf{E}_{i j}\right)$.

Notice that we do not use all $D^{2}$ entries of $M$. For each player $i$, we use $\sum_{k \in I-\{i\}} d_{k}$ rows and $d_{i}$ columns of $M$. Thus we could just use a totally nonsingular matrix with $D-\min _{i \in I} d_{i}$ rows and $\max _{i \in I} d_{i}$ columns.

For example, for a game of 3 players with 3 pure strategies each, using the above $6 \times 6$ totally nonsingular matrix, we arrive at the system:

$$
\begin{aligned}
\left(\sigma_{21}+2 \sigma_{22}-1\right)\left(\sigma_{31}+2 \sigma_{32}-1\right) & =0, \\
\left(2 \sigma_{21}-4 \sigma_{22}-1\right)\left(2 \sigma_{31}-4 \sigma_{32}-1\right) & =0, \\
\left(4 \sigma_{11}+16 \sigma_{12}-1\right)\left(4 \sigma_{31}+16 \sigma_{32}-1\right) & =0, \\
\left(8 \sigma_{11}-32 \sigma_{12}-1\right)\left(8 \sigma_{31}-32 \sigma_{32}-1\right) & =0, \\
\left(16 \sigma_{11}+128 \sigma_{12}-1\right)\left(16 \sigma_{21}+128 \sigma_{22}-1\right) & =0, \\
\left(32 \sigma_{11}-256 \sigma_{12}-1\right)\left(32 \sigma_{21}-256 \sigma_{22}-1\right) & =0 .
\end{aligned}
$$

If we replaced the 1 in each factor by $\sum_{j_{k}=1}^{d_{k}} \sigma_{k j_{k}}$ and expanded out the polynomials, we could determine for which payoff functions this is the system $\left(\mathbf{E}_{i j}\right)$. For instance, in the second equation the coefficient of $\sigma_{22} \sigma_{31}$ becomes -9 , so this says $u_{1}\left(s_{12}, s_{22}, s_{31}\right)-u_{1}\left(s_{10}, s_{22}, s_{31}\right)=-9$. The coefficient of $\sigma_{20} \sigma_{30}$ becomes 1 , so this says $u_{1}\left(s_{12}, s_{20}, s_{30}\right)-u_{1}\left(s_{10}, s_{20}, s_{30}\right)=1$. With this information we can construct games for which this is the system $\left(\mathbf{E}_{i j}\right)$.

Now to find all the solutions to this system, we define a $D \times D$ matrix $P$ by $P_{n(i, j) n(k, l)}=0$ if $i=k$ and $P_{n(i, j) n(k, l)}=1$ if $i \neq k$. In the example of a game with 
3 players, each with 3 pure strategies, we have

$$
P=\left[\begin{array}{llllll}
0 & 0 & 1 & 1 & 1 & 1 \\
0 & 0 & 1 & 1 & 1 & 1 \\
1 & 1 & 0 & 0 & 1 & 1 \\
1 & 1 & 0 & 0 & 1 & 1 \\
1 & 1 & 1 & 1 & 0 & 0 \\
1 & 1 & 1 & 1 & 0 & 0
\end{array}\right]
$$

We associate the $n(i, j)$ th row with the equation $\left(\mathbf{E}_{i j}\right)$ and the $n(i, j)$ th column with the variable $\sigma_{i j}$. So an entry of $P$ is 1 if and only if the corresponding variable appears in the corresponding equation.

The reader is familiar with the determinant of a matrix, which is the sum of certain signed products of entries of the matrix. The permanent of a matrix is the sum of those same products of entries of the matrix, but without the signs. In other words, for a $D \times D$ matrix $P$, the permanent of $P$ is the sum over all permutations $\tau$ of $1, \ldots, D$ of the products $\prod_{n=1}^{D} P_{n \tau(n)}$.

To find a solution of $(\mathbf{S})$, we pick $D$ entries of $P$ whose product contributes 1 to the permanent of $P$. In other words, we pick a permutation $\tau$ of $1, \ldots, D$ such that $\prod_{n=1}^{D} P_{n \tau(n)}=1$. For example, the italicized entries below represent such a choice:

$$
\left[\begin{array}{llllll}
0 & 0 & 1 & 1 & 1 & 1 \\
0 & 0 & 1 & 1 & 1 & 1 \\
1 & 1 & 0 & 0 & 1 & 1 \\
1 & 1 & 0 & 0 & 1 & 1 \\
1 & 1 & 1 & 1 & 0 & 0 \\
1 & 1 & 1 & 1 & 0 & 0
\end{array}\right]
$$

Now if $\tau(n(i, j))=n(k, l)$, this tells us to make equation $\left(\mathbf{E}_{i j}\right)$ hold by setting the factor $1+\sum_{j_{k}=1}^{d_{k}} \lambda_{k j_{k}}^{(i j)} \sigma_{k j_{k}}$ equal to zero. In the above example, the above choice tells us to set:

$$
\begin{aligned}
\sigma_{31}+2 \sigma_{32}-1 & =0, \\
2 \sigma_{21}-4 \sigma_{22}-1 & =0, \\
4 \sigma_{11}+16 \sigma_{12}-1 & =0, \\
8 \sigma_{31}-32 \sigma_{32}-1 & =0, \\
16 \sigma_{11}+128 \sigma_{12}-1 & =0, \\
32 \sigma_{21}-256 \sigma_{22}-1 & =0 .
\end{aligned}
$$

Now we have a system of $d_{i}$ linear equations in the $d_{i}$ variables $\sigma_{i j}$, for each $i$. Since we chose the coefficients from a totally nonsingular matrix, each system of $d_{i}$ linear 
equations has a unique solution. In this case, we find:

$$
\sigma_{11}=\frac{7}{16}, \quad \sigma_{12}=-\frac{3}{64}, \quad \sigma_{21}=\frac{21}{32}, \quad \sigma_{22}=\frac{5}{64}, \quad \sigma_{31}=\frac{17}{24}, \quad \sigma_{32}=\frac{7}{48} .
$$

(Clearly this particular solution does not satisfy the nonnegativity constraints, which we would also have to check if we were interested in the Nash equilibria of this particular game.)

Notice that this procedure would give us the same set of equations multiple times. For example, the choice of $D$ other entries in $P$ represented by the italicized entries below:

$$
\left[\begin{array}{llllll}
0 & 0 & 1 & 1 & 1 & 1 \\
0 & 0 & 1 & 1 & 1 & 1 \\
1 & 1 & 0 & 0 & 1 & 1 \\
1 & 1 & 0 & 0 & 1 & 1 \\
1 & 1 & 1 & 1 & 0 & 0 \\
1 & 1 & 1 & 1 & 0 & 0
\end{array}\right]
$$

gives the same system of equations. The problem is that for each set of $d_{i}$ columns corresponding to the variables $\sigma_{i 1}, \ldots, \sigma_{i d_{i}}$, we can apply any permutation to that set of columns without affecting the meaning of our choice. So if we carried out this procedure naïvely, it would repeat each solution $\prod_{i \in I} d_{i}$ ! times. We should avoid solving the same system twice. However, if we obtain the same solution from a different choice of which factor in each of the equations to set to zero, then we should perturb our totally nonsingular matrix so this doesn't happen, for reasons which will become clear later.

Carrying out this procedure, we find all 10 roots of $(\mathbf{E})$. We list the values of $\left(\sigma_{11}, \sigma_{12}, \sigma_{21}, \sigma_{22}, \sigma_{31}, \sigma_{32}\right)$ below, along with the corresponding permutations of $1, \ldots, D$ :

$$
\begin{array}{rr}
\left(\frac{3}{64}, \frac{1}{512}, \frac{3}{4}, \frac{1}{8}, \frac{3}{16}, \frac{1}{64}\right), & 5,6,1,2,3,4 \\
\left(\frac{7}{32}, \frac{3}{128}, \frac{21}{16}, \frac{-5}{32}, \frac{5}{12}, \frac{-1}{24}\right), & 4,6,1,5,2,3 \\
\left(\frac{17}{96}, \frac{7}{384}, \frac{21}{16}, \frac{-5}{32}, \frac{7}{8}, \frac{3}{16}\right), & 3,6,1,5,2,4 \\
\left(\frac{5}{48}, \frac{-1}{192}, \frac{129}{160}, \frac{31}{320}, \frac{5}{12}, \frac{-1}{24}\right), & 4,5,1,6,2,3 \\
\left(\frac{7}{16}, \frac{-3}{64}, \frac{129}{160}, \frac{31}{320}, \frac{7}{8}, \frac{3}{16}\right), & 3,5,1,6,2,4 \\
\left(\frac{7}{32}, \frac{3}{128}, \frac{33}{80}, \frac{-7}{160}, \frac{7}{4}, \frac{-3}{8}\right), & 4,6,2,5,1,3 \\
\left(\frac{17}{96}, \frac{7}{384}, \frac{33}{80}, \frac{-7}{160}, \frac{17}{24}, \frac{7}{48}\right), & 3,6,2,5,1,4
\end{array}
$$




$$
\begin{array}{ll}
\left(\frac{5}{48}, \frac{-1}{192}, \frac{21}{32}, \frac{5}{64}, \frac{7}{4}, \frac{-3}{8}\right), & 4,5,2,6,1,3 \\
\left(\frac{7}{16}, \frac{-3}{64}, \frac{21}{32}, \frac{5}{64}, \frac{17}{24}, \frac{7}{48}\right), & 3,5,2,6,1,4 \\
\left(\frac{3}{16}, \frac{1}{64}, \frac{3}{64}, \frac{1}{512}, \frac{3}{4}, \frac{1}{8}\right), & 3,4,5,6,1,2
\end{array}
$$

We note that the first and last of these satisfy the nonnegativity constraints, and hence are Nash equilibria. (The reader may notice some symmetries between pairs of the solutions. This is because we happened to use the same region of $M$ for all the players.)

We can find the rest of the solutions of the system $(*)$ corresponding to this same game too. Suppose we require $v_{i j}$ to be positive instead of vanishing. Then the variable $\sigma_{i j}$ goes away from every equation and the equation $\left(\mathbf{E}_{i j}\right)$ is replaced by the equation $\sigma_{i j}=0$. In the above example, suppose we require $v_{31}$ to be positive. Then we obtain a new system

$$
\begin{aligned}
\left(\sigma_{21}+2 \sigma_{22}-1\right)\left(2 \sigma_{32}-1\right) & =0, \\
\left(2 \sigma_{21}-4 \sigma_{22}-1\right)\left(4 \sigma_{32}-1\right) & =0, \\
\left(4 \sigma_{11}+16 \sigma_{12}-1\right)\left(16 \sigma_{32}-1\right) & =0, \\
\left(8 \sigma_{11}-32 \sigma_{12}-1\right)\left(32 \sigma_{32}-1\right) & =0, \\
\sigma_{31} & =0, \\
\left(32 \sigma_{11}-256 \sigma_{12}-1\right)\left(32 \sigma_{21}-256 \sigma_{22}-1\right) & =0 .
\end{aligned}
$$

This system corresponds to the $n(i, j), n(i, j)$ minor of $M$, which is also totally nonsingular. We can use the same matrix $P$, but zero out the $n(i, j)$ th row and $n(i, j)$ th column and set the $n(i, j), n(i, j)$ entry to 1 . In our example:

$$
P_{i j}^{\prime}=\left[\begin{array}{llllll}
0 & 0 & 1 & 1 & 0 & 1 \\
0 & 0 & 1 & 1 & 0 & 1 \\
1 & 1 & 0 & 0 & 0 & 1 \\
1 & 1 & 0 & 0 & 0 & 1 \\
0 & 0 & 0 & 0 & 1 & 0 \\
1 & 1 & 1 & 1 & 0 & 0
\end{array}\right]
$$

Now we follow the same procedure as above, performing the cofactor expansion of the permanent along the $n(i, j)$ th row. For example, the product of italicized entries 
below contributes 1 to the permanent of this matrix:

$$
\left[\begin{array}{llllll}
0 & 0 & 1 & 1 & 0 & 1 \\
0 & 0 & 1 & 1 & 0 & 1 \\
1 & 1 & 0 & 0 & 0 & 1 \\
1 & 1 & 0 & 0 & 0 & 1 \\
0 & 0 & 0 & 0 & 1 & 0 \\
1 & 1 & 1 & 1 & 0 & 0
\end{array}\right]
$$

The corresponding system is:

$$
\begin{aligned}
\sigma_{21}+2 \sigma_{22}-1 & =0, \\
2 \sigma_{21}-4 \sigma_{22}-1 & =0, \\
4 \sigma_{11}+16 \sigma_{12}-1 & =0, \\
32 \sigma_{32}-1 & =0, \\
\sigma_{31} & =0, \\
32 \sigma_{11}-256 \sigma_{12}-1 & =0 .
\end{aligned}
$$

Its solution is:

$$
\begin{aligned}
& \sigma_{11}=\frac{17}{96}, \quad \sigma_{12}=\frac{7}{384}, \\
& \sigma_{21}=\frac{3}{4}, \quad \sigma_{22}=\frac{1}{8}, \\
& \sigma_{31}=0, \quad \sigma_{32}=\frac{1}{32} .
\end{aligned}
$$

This at least satisfies the nonnegativity constraints on the $\sigma_{i j}$ 's. If we are interested in the Nash equilibria of this game, we also have to check that it satisfies the nonnegativity constraints on the $v_{i j}$ 's, namely, that $v_{31} \geq 0$. We substitute the $\sigma_{i j}$ 's into

$$
\left(16 \sigma_{11}+128 \sigma_{12}-1\right)\left(16 \sigma_{21}+128 \sigma_{22}-1\right),
$$

the expected payoff to player 3 from playing $\sigma_{31}$, obtaining $225 / 2$, which is strictly greater than zero. So this solution to the polynomial system is not a Nash equilibrium, since $s_{31}$ is a strictly better response to $\sigma_{-3}$ than the value of $\sigma_{3}$ given by this solution.

In this way we see that our specially constructed factorizable game of a given format $\left(N ; d_{1}, \ldots, d_{N}\right)$ contains subgames of every smaller format $\left(N^{\prime}: d_{1}^{\prime}, \ldots, d_{N^{\prime}}^{\prime}\right)$, with $N^{\prime} \leq N$ and $d_{i}^{\prime} \leq d_{i}$ for each $i$, such that the subgames are also factorizable. As we will see, we only need to solve one (or a few) of the factorizable polynomial systems for each format in the manner we have described so far.

Geometrically, by constructing the $D \times D$ totally nonsingular matrix $M$, we picked $D$ vectors in $D$-dimensional space, such that not only are these all distinct points, but if we project any $m$ of them onto any $m$-dimensional coordinate subspace, the images 
are all also distinct. The condition that two particular such images coincide is an equation, which is satisfied only on a subset of real $D$-dimensional space of strictly lower dimension. So every open subset of real $D$-dimensional space does not satisfy the condition, almost everywhere. Since there are only finitely many of these conditions, every open subset does not satisfy any of them, almost everywhere. In particular, we could construct a totally nonsingular matrix such that all the nonnegativity constraints of our factorizable game also held with strict inequality. However, while this would provide an example of a game with the maximal possible number of totally mixed Nash equilibria, it would not be particularly relevant to the use we will be making of our specially constructed factorizable games.

\section{Polyhedra and polynomial systems}

In this section, we explain how polyhedra are associated to polynomial systems. The combinatorics of the associated polyhedra will enable us to count the number of solutions of a generic polynomial system. The game-theoretic case is a particularly beautiful and clear example of this relationship, but the reader may skip this section without loss.

With a system of polynomial equations is associated a polyhedral subdivision, that is, a polyhedron which is subdivided into cells, each of which is also a polyhedron, glued together along their faces. We illustrate this for a game of 3 players with 2 pure strategies each, since in this case the polyhedral subdivision is 3-dimensional. Using the same totally nonsingular matrix, we obtain the following system of factored equations:

$$
\begin{gathered}
\left(\sigma_{21}-1\right)\left(\sigma_{31}-1\right)=0, \\
\left(2 \sigma_{11}-1\right)\left(2 \sigma_{31}-1\right)=0, \\
\left(4 \sigma_{11}-1\right)\left(4 \sigma_{21}-1\right)=0 .
\end{gathered}
$$

Expanding this out, we obtain

$$
\begin{gathered}
\sigma_{21} \sigma_{31}-\sigma_{21}-\sigma_{31}+1=0, \\
4 \sigma_{11} \sigma_{31}-2 \sigma_{11}-2 \sigma_{31}+1=0, \\
16 \sigma_{11} \sigma_{21}-4 \sigma_{11}-4 \sigma_{21}+1=0 .
\end{gathered}
$$

A monomial $x_{1}^{\alpha_{1}} x_{2}^{\alpha_{2}} \cdots x_{n}^{\alpha_{n}}$ in $n$ variables can be represented by the lattice point $\left(\alpha_{1}, \ldots, \alpha_{n}\right) \in \mathbb{N}^{n}$ of its exponents. For example, the lattice of monomials in two variables $x$ and $y$ is depicted in Fig. 2.

The Newton polytope of a polynomial equation is the convex hull of the lattice points of the monomials occuring in that equation. In our example system in 3 variables $\sigma_{11}, \sigma_{21}$, and $\sigma_{31}$, the Newton polytope of Eq. 4 is depicted in Fig. 3, the Newton polytope of Eq. 5 is depicted in Fig. 4, and the Newton polytope of Eq. 6 is depicted in Fig. 5. 
Fig. 2 The monomial lattice in two variables
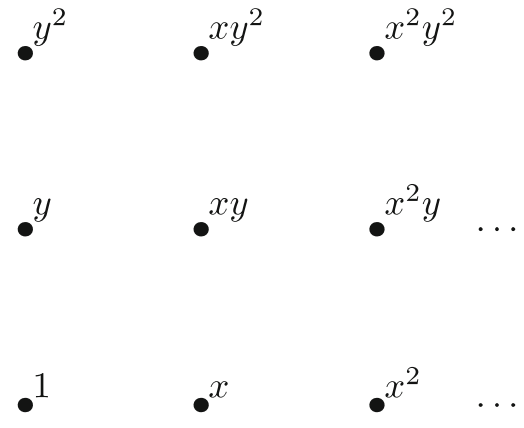

Fig. 3 The Newton polytope of $\sigma_{21} \sigma_{31}-\sigma_{21}-\sigma_{31}+1=0$

Fig. 4 The Newton polytope of $4 \sigma_{11} \sigma_{31}-2 \sigma_{11}-2 \sigma_{31}+1=0$
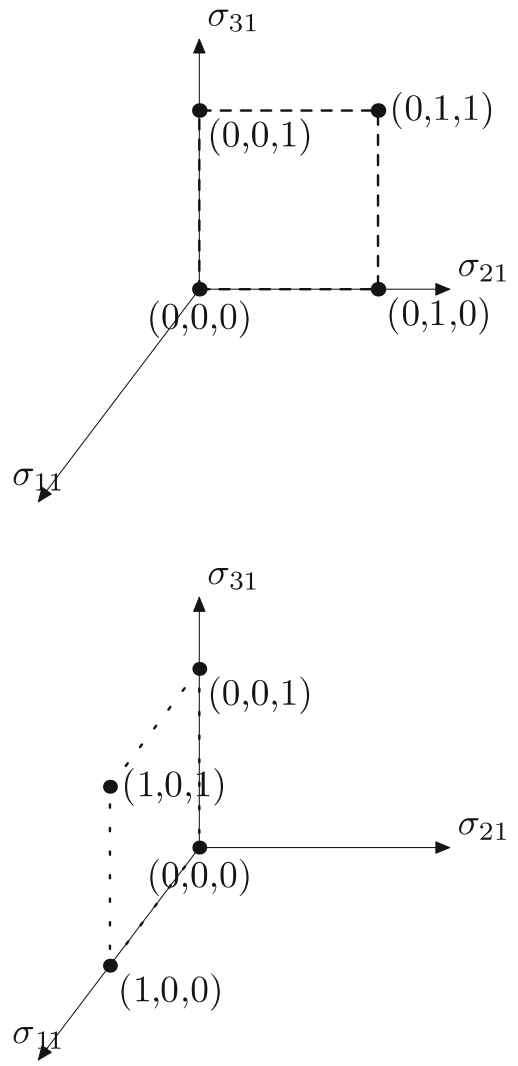

The Minkowski sum of an $n$-dimensional polytope with vertices $V_{01}, \ldots, V_{0 m_{0}}$ and an $n$-dimensional polytope with vertices $V_{11}, \ldots, V_{1 m_{1}}$ is the convex hull of the points $V_{0 i}+V_{1 j}$ in $n$-dimensional space, for $i=1, \ldots, m_{0}$ and $j=1, \ldots, m_{1}$. Figure 6 depicts the Minkowski sum of the Newton polytopes of Eqs. 4 and 5. We can think of the Minkowski sum as translating one of the polytopes along each edge of the other 
Fig. 5 The Newton polytope of $16 \sigma_{11} \sigma_{31}-4 \sigma_{11}-4 \sigma_{21}+1=0$

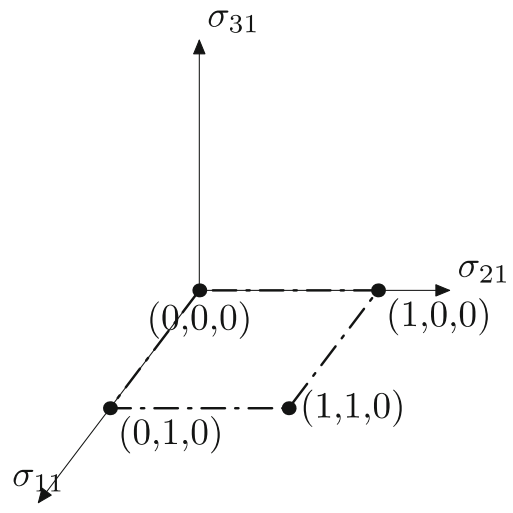

Fig. 6 Minkowski sum of a pair of Newton polytopes

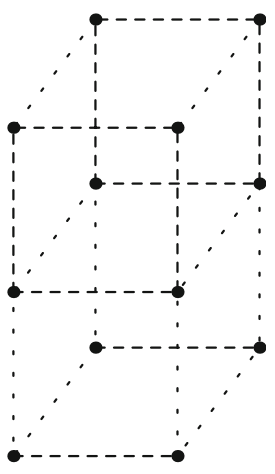

polytope. Here we have colored the edges as if we first translated the dotted polytope along each edge of the dashed polytope. The dotted edges came from the original dotted polytope, and the dashed edges came from edges of the dashed polytope along which we translated. Notice that we can do this in more than one way. For example, we could have colored the edges as if we translated the dashed polytope along each edge of the dotted polytope first. Such a coloring of the Minkowski sum gives us a mixed subdivision, which in this case has two cells, the two cubes in Fig. 6.

Finally, the Minkowski sum of all three of our Newton polytopes is depicted in Fig. 7. A cell of a mixed subdivision is mixed if each color delineates only edges (or possibly vertices) in the cell, not higher-dimensional faces. In Fig. 7, the top left front cube is not mixed, because two of its faces are dashed squares, and the top right front cube is not mixed, because two of its faces are dotted-and-dashed squares. We see that two of the cells in this mixed subdivision are mixed. Each mixed cell tells us how to obtain certain solutions to the factorizable polynomial system. Namely, in each polynomial equation, we should look at the edges with the corresponding color in the mixed cell, and set the factor(s) corresponding to the directions of those edges to zero. In the game-theoretic case, there will be exactly one solution corresponding to each mixed cell, since the polynomial system has degree at most 1 in any variable.

For example, Fig. 8 depicts the bottom mixed cell of the mixed subdivision. To find the solution corresponding to this mixed cell, we should set the factor $\sigma_{21}-1$ to zero 
Fig. 7 Minkowski sum of three newton polytopes

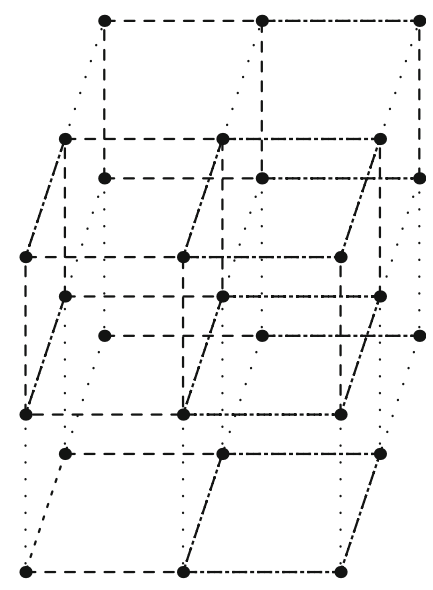

Fig. 8 One mixed cell of the mixed subdivision

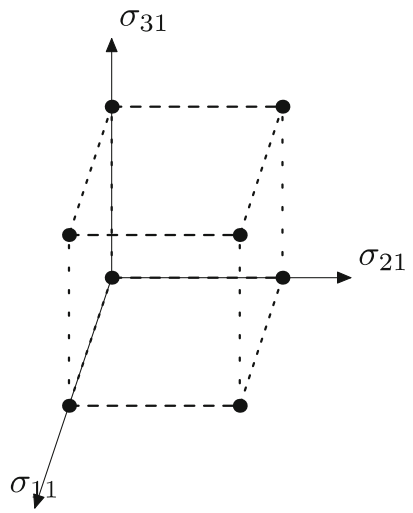

in Eq. 1; we should set the factor $2 \sigma_{31}-1$ to zero in Eq. 2 ; and we should set the factor $4 \sigma_{11}-1$ to zero in Eq. 3. This gives us the solution

$$
\sigma_{11}=\frac{1}{4}, \quad \sigma_{21}=1, \quad \sigma_{31}=\frac{1}{2}
$$

Figure 9 depicts the top mixed cell of the mixed subdivision. To find the solution corresponding to this mixed cell, we should set the factor $\sigma_{31}-1$ to zero in Eq. 1; we should set the factor $2 \sigma_{11}-1$ to zero in Eq. 2 ; and we should set the factor $4 \sigma_{21}-1$ to zero in Eq. 3. This gives us the solution

$$
\sigma_{11}=\frac{1}{2}, \quad \sigma_{21}=\frac{1}{4}, \quad \sigma_{31}=1
$$


Fig. 9 Another mixed cell of the mixed subdivision

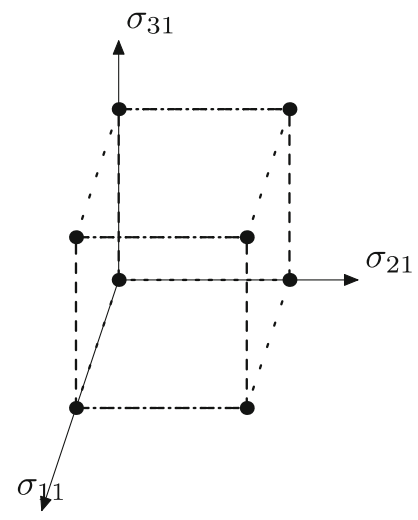

In the game-theoretic case, the cells of the mixed subdivision are always $D$-dimensional cubes (or hypercubes). The matrix $P$ in this example is

$$
\left[\begin{array}{lll}
0 & 1 & 1 \\
1 & 0 & 1 \\
1 & 1 & 0
\end{array}\right]
$$

Recall that the rows correspond to equations, and the columns correspond to variables. A permutation which contributes to the permanent will tell us which equation corresponds to each variable. The edges of the mixed cube going in the direction corresponding to that variable will correspond with that equation, and the linear factor containing that variable in that equation will be set to zero.

\section{Finding all Nash equilibria by polyhedral homotopy continuation}

In this section, we turn to the solution of generic games. We have taken considerable trouble to find all Nash equilibria of one particular kind of specially constructed game. The good news is that once we have done this for one particular game format, we can easily solve any generic game of that format. ${ }^{2}$ Furthermore, we can even more easily look for one or a few of the Nash equilibria, look for Nash equilibria with some particular small support, and so forth. The key idea is to "morph" the specially constructed polynomial system into the polynomial system we are actually interested in. As we do this, the solutions to $(\mathbf{E})$ for the specially constructed game will also morph into solutions to $(\mathbf{E})$ for the game of interest. Moreover, this procedure is "embarrassingly parallel". The morphing of each solution is independent of the morphing of every other solution. We can partition the solutions to $(\mathbf{E})$ for the specially constructed game into

\footnotetext{
2 It is not quite true that we only need to solve one game of each format. Something untoward could happen on the way from this game to the one we are interested in; e.g., we could run into a game whose set of Nash equilibria is positive-dimensional. So we should have the solutions to a few of the specially constructed games ready to hand. We can make more such games by using different regions of our totally nonsingular matrix.
} 
subsets, and hand each subset to a different processor. If somewhere along the way a morphed solution begins to look disappointing (for example, it doesn't look like it will end up satisfying the nonnegativity constraints, or it starts to have an imaginary component that we fear won't go away), we can always stop morphing that solution and come back to it later if more promising ones do not pan out.

We should emphasize that the techniques we are describing apply to generic games. A generic game has a finite number of Nash equilibria, by the generic finiteness theorem of Harsanyi (1973). On the other hand, it is possible to construct games whose set of Nash equilibria includes the set of solutions to any arbitrary polynomial system with real coefficients (Datta 2003b). This set may be a curve, a surface, or a higherdimensional manifold. In this case, solving the polynomial system would be much more complicated than the techniques we have described. We refer the reader to the text (Basu et al. 2003) on solving polynomial systems with real coefficients.

Readers may be familiar with the homotopy continuation method under the guise of "tracing procedures", such as the techniques of Lemke-Howson or Govindan and Wilson. To solve a polynomial system by the homotopy continuation method, we create a family $\mathcal{F}$ of polynomial systems $f_{1 t}=0, f_{2 t}=0, \ldots, f_{m t}=0$ parametrized by a variable $t$ lying in $[0,1]$, such that the polynomial system we want to solve is $f_{11}=$ $0, f_{21}=0, \ldots, f_{m 1}=0$, and the polynomial system $f_{10}=0, f_{20}=0, \ldots, f_{m 0}=0$, called the starting system, is easy to solve. We consider each polynomial system in $\mathcal{F}$ to lie in some topological space such that for any particular point, the map of the polynomial space which evaluates the polynomial at that point is continuous. We require the map from $[0,1]$ to this space to be continuous, or in other words a homotopy. Now suppose $\left(x_{1}, \ldots, x_{n}\right)$ satisfies the polynomial system $f_{1 t_{0}}, \ldots, f_{m t_{0}}$, and $t_{1}$ is near $t_{0}$. Since the homotopy is continuous, $f_{1 t_{1}}, \ldots, f_{m t_{1}}$ must be near $f_{1 t_{0}}, \ldots, f_{m t_{0}}$, and so $f_{1 t_{1}}\left(x_{1}, \ldots, x_{n}\right)$ must lie near zero, $f_{2 t_{1}}\left(x_{1}, \ldots, x_{n}\right)$ must lie near zero, and so forth. Therefore, since polynomial functions are also continuous, we can look for a root of the system $f_{1 t_{1}}, \ldots, f_{m t_{1}}$ near $\left(x_{1}, \ldots, x_{n}\right)$. We make a prediction, i.e., we guess a possible root of $f_{1 t_{1}}, \ldots, f_{m t_{1}}$ near $\left(x_{1}, \ldots, x_{n}\right)$, and then a correction, i.e., we find an actual root near our guess, using Newton's method for example. Once we have one, we can proceed to the next iteration for $t_{2}$ near $t_{1}$, and so forth. At the end we will have a path from our original root $\left(x_{1}, \ldots, x_{n}\right)_{t=0}$ to a root $\left(x_{1}, \ldots, x_{n}\right)_{t=1}$ of the desired system. Herings and Peeters (2009) presents a survey of previous uses of homotopy continuation methods in game theory. The book (Sommese and Wampler 2005) gives a recent survey of numerical methods for solving polynomial systems, including detailed treatment of homotopy continuation, and in particular polyhedral homotopy continuation.

If we fix the number of equations, and the Newton polytopes of each equation, then the set of such polynomial systems becomes a vector space over the coefficient field. Each monomial occurring in each equation corresponds to a basis element of this vector space, and a particular polynomial system is uniquely specified by giving the coefficients of all the monomials in all the equations. In particular, if the coefficient field is $\mathbb{R}$ then this space of polynomial systems is a finite-dimensional real vector space. Hence, it is equipped with a topology, the usual topology of such spaces. We will call the number of equations together with the Newton polytopes of each equation the shape of a polynomial system. Polyhedral homotopy continuation is 
simply homotopy continuation among polynomial systems of the same shape. The word "polyhedral" refers to the polyhedral subdivision introduced in the previous section.

The Bernstein-Kouchnirenko theorem (Bernstein 1975; Kouchnirenko 1976) tells us that the number of 0 -dimensional complex roots, none of whose components are zero, of every generic polynomial system of a given shape is the same. This number is called the Bernstein number of the system. Polyhedral homotopy continuation (Huber and Sturmfels 1995) provides an alternate constructive proof of this fact. Thus, if we apply polyhedral homotopy continuation to a generic polynomial system we will find a unique (possibly complex) root of the system in question at the end of each path leading from one of the roots of the starting system. McKelvey and McLennan (1997) applied the Bernstein-Kouchnirenko theorem to find the number of complex roots of the polynomial system $(\mathbf{E})$ for a generic game: it is the permanent of the matrix $P$, divided by $\prod_{i \in I} d_{i} !$. Furthermore, they constructed games in which all these roots are actual Nash equilibria, i.e., they are real and nonnegative. In Datta (2003a) we generalized this theorem to polynomial systems obeying special conditions, such as those arising from graphical games, i.e., games in which the payoffs to some players depend only on the strategies chosen by a subset of the other players. Briefly put, the special conditions imply that some of the entries in $P$ are zero. This means that the polynomial system will have a smaller number of roots (in some cases, none), and thus leads to a tighter upper bound on the number of totally mixed Nash equilibria for such games.

In general, the most difficult part of polyhedral homotopy continuation is computing the mixed subdivision, finding a polynomial system which is generic, and using the mixed subdivision to find all the roots. Once all this is done, we can follow a simple linear homotopy (i.e., of the form $f_{t}=\left(1-t^{k}\right) f_{0}+t^{k} f_{1}$ for some $k \geq 1$ ) from this starting system to the desired system, which is relatively straightforward. Therefore polyhedral homotopy continuation is particularly well-suited in the case of game theory, since we know exactly how to find and solve a starting system. The system $\left(\mathbf{E}_{i j}\right)$ is an example of a multihomogeneous system. Every monomial appearing in one of these equations has the same degree, namely 1, in all of player 1's variables put together, the same degree, namely 1 , in all of player 2's variables put together, and so forth. In the same way multihomogeneous systems are generally easy to solve by polyhedral homotopy continuation. As we saw, adding in the conditions $\left(\mathbf{E}_{i 0}\right)$ does not make the problem more difficult, although it does mean multihomogeneity no longer holds. The system (E) is a linear product family, as described in Section 8.4.3 of Sommese and Wampler (2005).

We may not even need to find all the roots of the starting system before starting to find the Nash equilibria of the desired game. Once we have a single root of the starting system, we can start tracing it. However, if we are unlucky the corresponding root of the desired game may not be nonnegative or even real, in which case we will have to go find another root of the starting system to trace. McLennan (2002) describes the expected number of real roots of a random multihomogeneous system of polynomial equations, and gives the lower bound as the square root of the Bernstein number (the number of complex roots of a generic game).

Let's expand out our factorizable polynomial system for the game of 3 players with 3 pure strategies each. 


$$
\begin{aligned}
& \sigma_{21} \sigma_{31}+2 \sigma_{21} \sigma_{32}+2 \sigma_{22} \sigma_{31}+4 \sigma_{22} \sigma_{32}-\sigma_{21}-2 \sigma_{22}-\sigma_{31}-2 \sigma_{32}+1=0, \\
& 4 \sigma_{21} \sigma_{31}-8 \sigma_{21} \sigma_{32}-8 \sigma_{22} \sigma_{31}+16 \sigma_{22} \sigma_{32}-2 \sigma_{21}+4 \sigma_{22}-2 \sigma_{31}+4 \sigma_{32}+1=0, \\
& 16 \sigma_{11} \sigma_{31}+64 \sigma_{11} \sigma_{32}+64 \sigma_{12} \sigma_{31}+256 \sigma_{12} \sigma_{32}-4 \sigma_{11}-16 \sigma_{12} \\
& \quad-4 \sigma_{31}-16 \sigma_{32}+1=0 \\
& 64 \sigma_{11} \sigma_{31}+256 \sigma_{11} \sigma_{32}+256 \sigma_{12} \sigma_{31}+1024 \sigma_{12} \sigma_{32} \\
& \quad-8 \sigma_{11}-32 \sigma_{12}-8 \sigma_{31}-32 \sigma_{32}+1=0, \\
& 256 \sigma_{11} \sigma_{21}+2048 \sigma_{11} \sigma_{22}+2048 \sigma_{12} \sigma_{21}+16384 \sigma_{12} \sigma_{22} \\
& \quad-16 \sigma_{11}-128 \sigma_{12}-16 \sigma_{21}-128 \sigma_{22}+1=0, \\
& 1024 \sigma_{11} \sigma_{21}-8192 \sigma_{11} \sigma_{22}-8192 \sigma_{12} \sigma_{21}+65536 \sigma_{12} \sigma_{22} \\
& -32 \sigma_{11}+256 \sigma_{12}-32 \sigma_{21}+256 \sigma_{22}+1=0 .
\end{aligned}
$$

We can make an input file for the polyhedral homotopy continuation software PHC (Verschelde 1999) which specifies this polynomial system. Since PHC orders the variables according to the order they have appeared in the file, we will write the equations in the reverse order from the system listed above, so that the variables will appear in the order $\sigma_{11}, \sigma_{12}, \sigma_{21}, \sigma_{22}, \sigma_{31}, \sigma_{32}$. Here is the input file game_of_3_x_3_x_3_ start_phc:

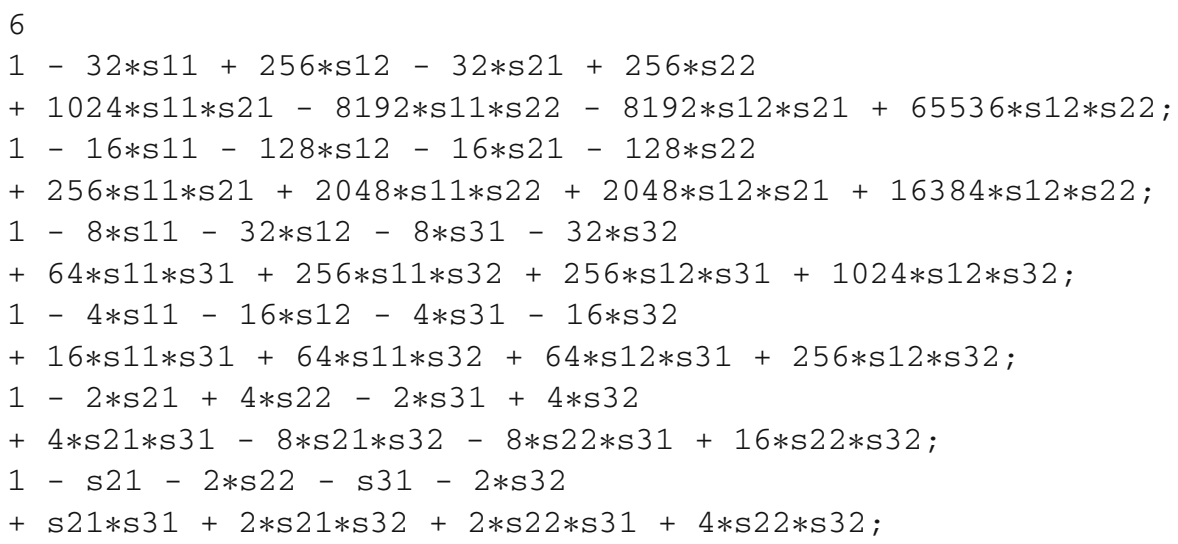

The first line specifies the number of equations, and the rest of the file specifies the equations. Since an unknown can only consist of up to 5 characters, we denote the variable $\sigma_{11}$ by s 11 in the file, and so forth.

We could find the roots of this factorizable system using PHC itself (in which case we should tell PHC about the linear product structure of our equations), or using other programs in the manner described above. We will list the 10 roots of the starting system we found before in another input file for PHC, which we call game_of_3_x_3_x_3_start_phc.roots. Here is the beginning of that file, including the first two roots:

\section{6}

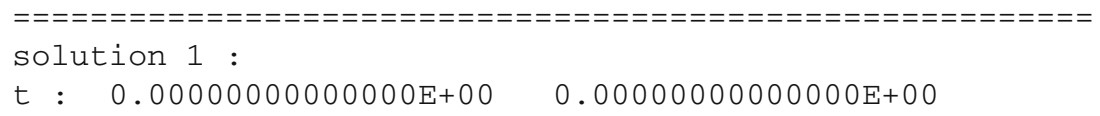




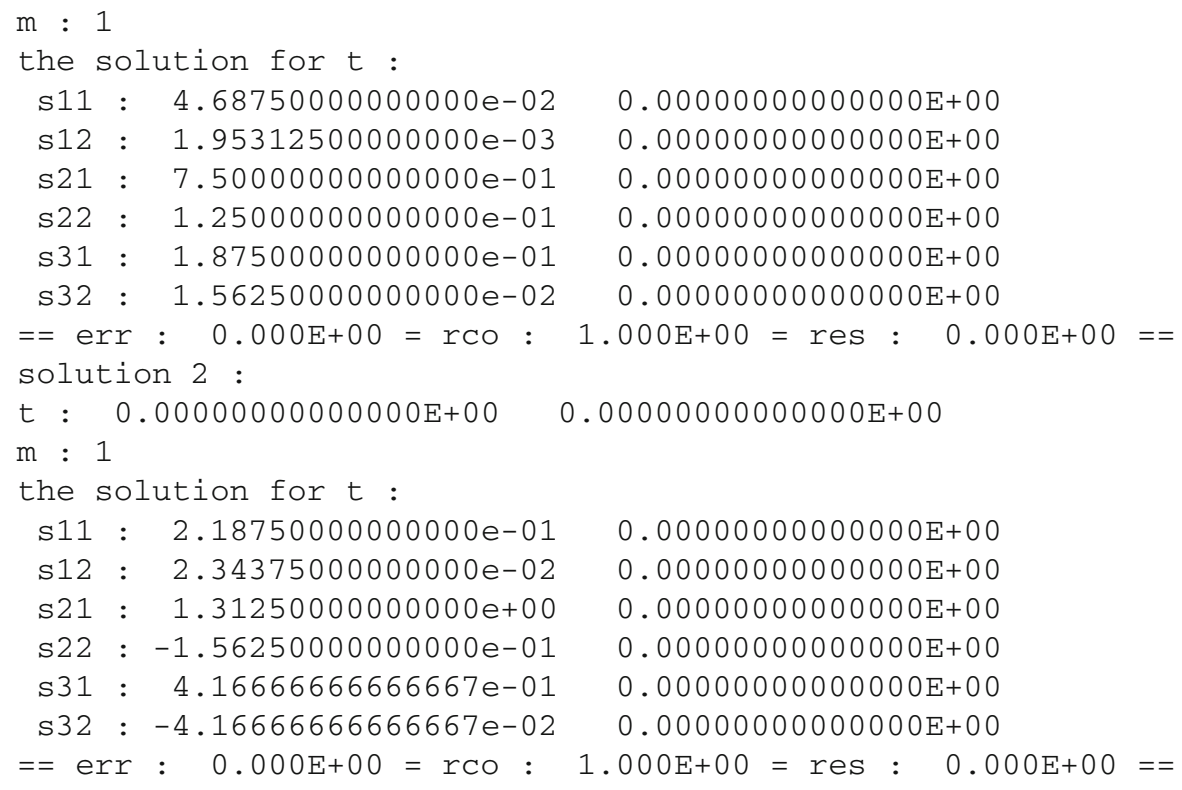

The first line indicates that the file contains 10 solutions in 6 unknowns. Here $t$ denotes the homotopy parameter, and $m$ denotes the multiplicity of each root. A line such as

\section{s11: $4.68750000000000 e-02 \quad 0.00000000000000 E+00$}

indicates that at this solution, the variable s11 has real part 0.046875 and imaginary part 0 . The lines

the solution for $t$ :

and

$==\operatorname{err}: 0.000 \mathrm{E}+00=$ rco: $1.000 \mathrm{E}+00=$ res $: 0.000 \mathrm{E}+00==$

are lines that would have been included by PHC if it had written this solution file itself, so we include them also even if we didn't use PHC to generate these solutions.

Finally, we write an input file game_of_3_x_3_x_3_phc with an example of another polynomial system of the same shape, which we would like to solve:

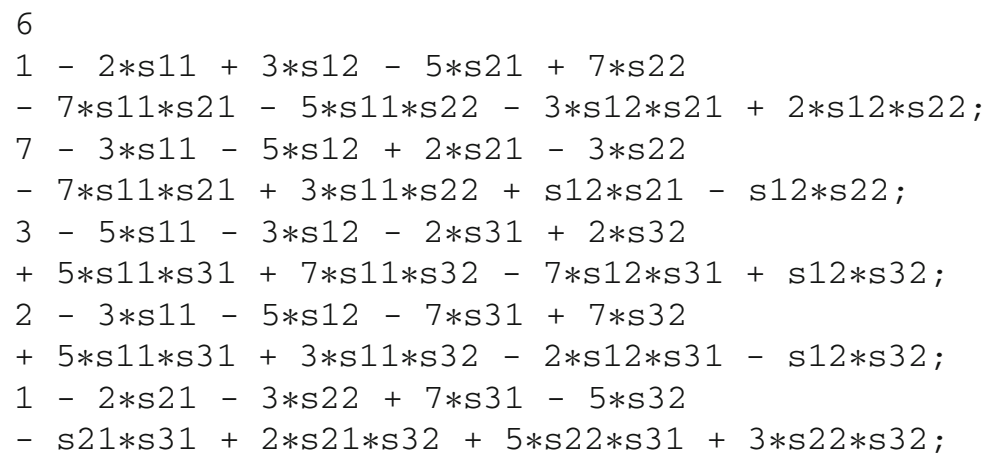


$1-\mathrm{s} 21+2 * \mathrm{~s} 22-3 * \mathrm{~s} 31-5 * \mathrm{~s} 32$

$+7 * \mathrm{~s} 21 * \mathrm{~s} 31-2 * \mathrm{~s} 21 * \mathrm{~s} 32+5 * \mathrm{~s} 22 * \mathrm{~s} 31+3 * \mathrm{~s} 22 * \mathrm{~s} 32 ;$

Now we can invoke PHC with the $-\mathrm{p}$ option, indicating that we already have a starting system and its solutions. Responding to the prompts, we specify that the target polynomial system is in the file game_of_3_x_3_x_3_phc, that we want PHC to write the output to a file game_of_3_x_3_x_3_phc.output and the solutions to a separate file game_of_3_x_3_x_3_phc.roots, and that we already have a start system in file game_of_3_x_3_x_3_start_phc with solutions in file game_of_3_x_3_x_3_start_phc.roots. At this point we are presented with several menus allowing us to change different options for controlling the homotopy continuation. At each point we can enter 0 to accept the default options. Finally we are presented with:

No more input expected. See output file for results.

and, possibly after some delay depending on how big our system is (in this case, there is no noticeable delay), the program exits. Now we can look at the solution file we specified game_of_3_x_3_x_3_phc.roots:

106

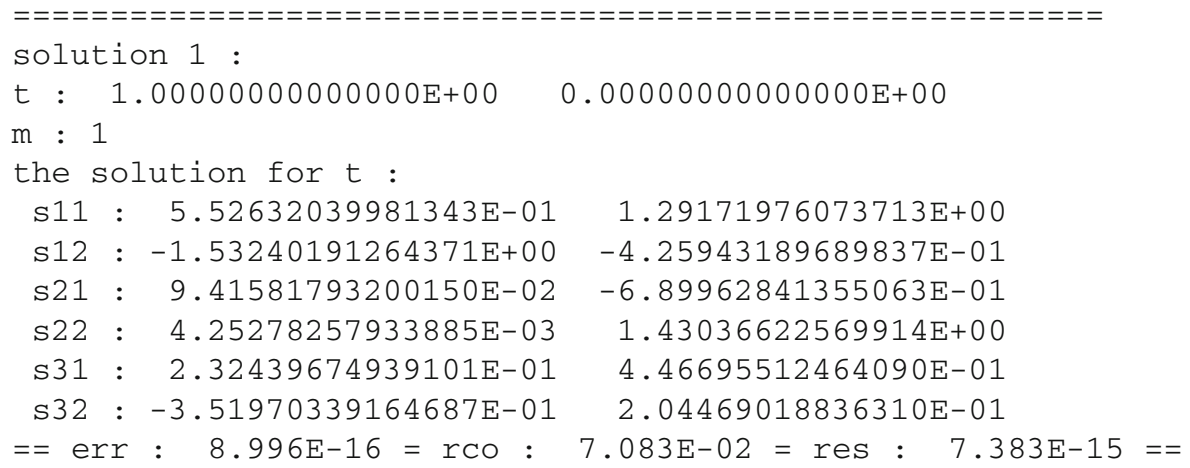

We see that each component of this solution has a nonzero imaginary part. So this solution is not of interest to us. Looking further down in the file, we see another solution:

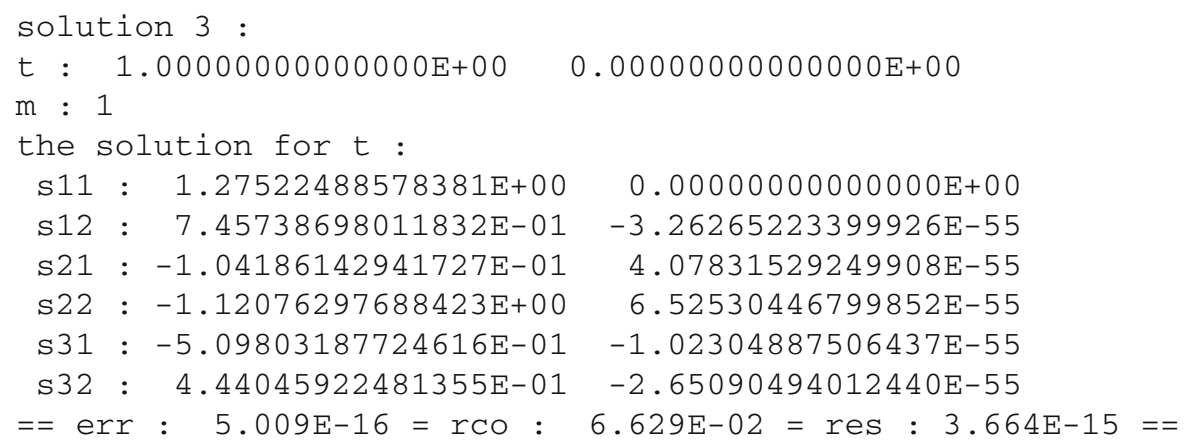

Here the imaginary parts occurring in the various components are very small, and could be due to numerical error. The eighth solution is similar. To test our hypothesis, we make another file game_of_3_x_3_x_3_phc.real_roots in which 
we include only these two roots, setting their imaginary parts to zero and renumbering them in sequence:

26

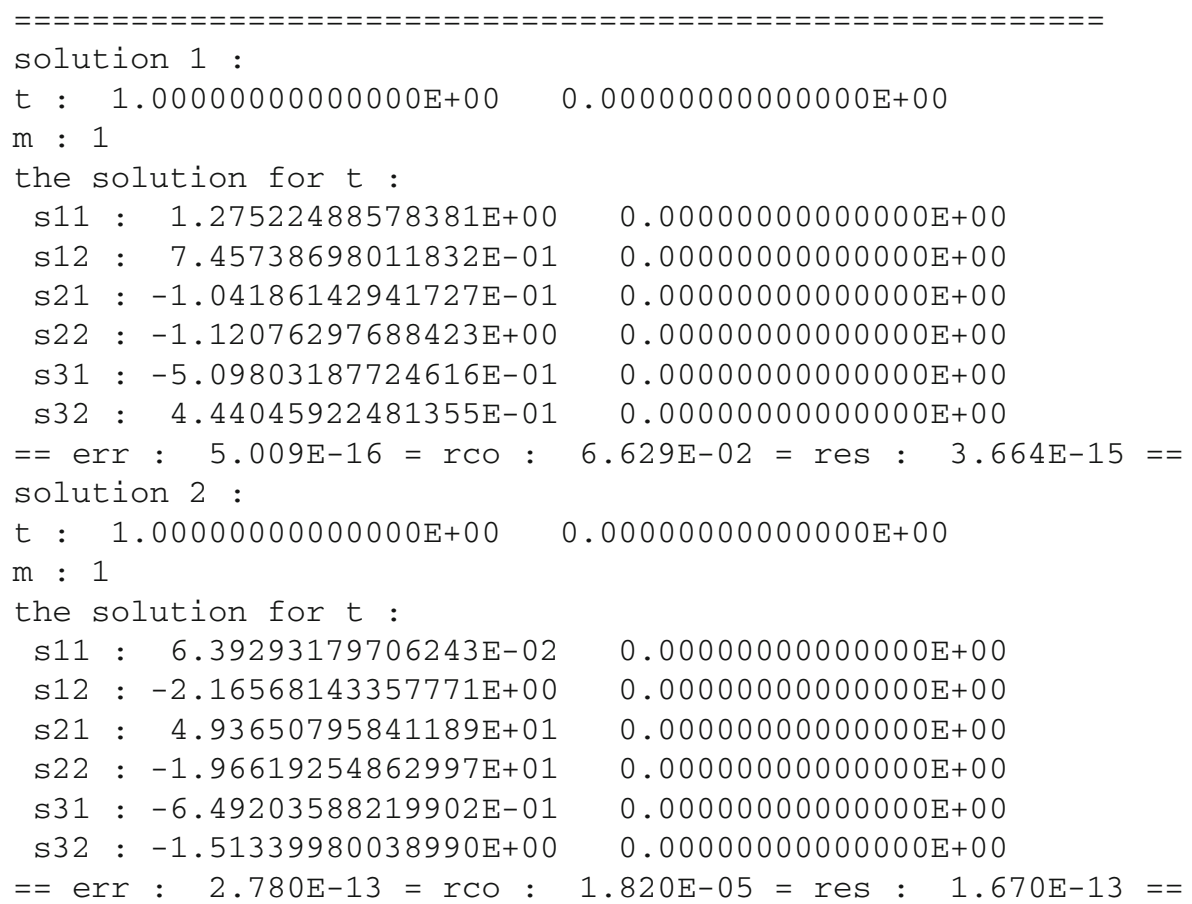

Then we ask PHC to validate them by calling it with the $-\mathrm{v}$ option, and selecting option 2 from the resulting menu, "Evaluation of the residuals using multi-precision arithmetic". This causes PHC to evaluate each polynomial system at our candidate roots, obtaining the residuals (the magnitudes of their images, which were supposed to vanish). We look in the file game_of_3_x_3_x_3_phc.validation_of_ real_roots for the section beginning THE RESIDUALS:

THE RESIDUALS with 16 decimal places :

residual $1: 1.2838672747 \mathrm{E}-14$

residual $2: 6.530598545 \mathrm{E}-13$

So we suspect that these roots are indeed real. Since they do not satisfy the nonnegativity constraints, they are not Nash equilibria. (Note well that even if all the components of a given solution were real and nonnegative, we would still have to check that $\mathrm{s} 11+\mathrm{s} 12<=1, \mathrm{~s} 21+\mathrm{s} 22<=1$, and s31 + s32<= 1.)

Thus, we can compute a library of starting systems for games of various formats. This initial computation may take a long time (indeed, computing the permanent is $N P$-hard), but only has to be done once (or a few times) for games of each format. Once we have done this, for any given game, we can look in the library for an appropriate starting system along with its roots, parcel out the roots among the processors we are using (possibly according to some heuristic scheme if we believe some of them 
are more likely to lead to Nash equilibria), and hand the starting system, the desired system, and the subset of roots to each processor.

If we wish, we can use interval computation along the path to each root to get a verified bound on where the final root is. However, interval techniques become computationally expensive in higher dimensions. For example, in $D$ dimensions an "interval" might be a box with $2^{D}$ corners.

\section{Solving polynomial systems using Gröbner bases}

So far we have discussed polyhedral homotopy continuation, which is predominantly a numerical method for solving particular polynomial systems. In this section, we discuss other, more symbolic methods of solving polynomial systems. Gröbner bases methods have great power to provide geometric insight and answer more general questions about games of a given format. We also briefly discuss resultants.

Recall that monomials correspond to points of the lattice $\mathbb{N}^{n}$. A monomial order is a total order $\preceq$ of $\mathbb{N}^{n}$, such that for any $\alpha, \beta, \gamma \in \mathbb{N}^{n}$, if $\alpha \preceq \beta$ then $\alpha+\gamma \preceq \beta+\gamma$. In other words, a monomial order is a total order which is compatible with addition of the points in $\mathbb{N}^{n}$, which corresponds to multiplication of monomials.

An example of a monomial order is the lexicographic order, which is defined as follows. First define some ordering on the variables, e.g., $x_{n} \preceq x_{n-1} \preceq \cdots \preceq x_{2} \preceq x_{1}$. Then the lexicographic order can be defined recursively on the number $n$ of variables: $x_{n}^{\alpha_{n}} \preceq x_{n}^{\beta_{n}}$ if and only if $\alpha_{n} \leq \beta_{n}$, and $x_{1}^{\alpha_{1}} x_{2}^{\alpha_{2}} \cdots x_{n}^{\alpha_{n}} \preceq x_{1}^{\beta_{1}} x_{2}^{\beta_{2}} \cdots x_{n}^{\beta_{n}}$ if and only if either $\alpha_{1} \leq \beta_{1}$, or $\alpha_{1}=\beta_{1}$ and $x_{2}^{\alpha_{2}} \cdots x_{n}^{\alpha_{n}} \preceq x_{2}^{\beta_{2}} \cdots x_{n}^{\beta_{n}}$.

Suppose we are given two polynomials in $n$ variables, $f_{1}\left(x_{1}, \ldots, x_{n}\right)$ and $f_{2}\left(x_{1}, \ldots\right.$, $\left.x_{n}\right)$. The set of monomials occurring with nonzero coefficients in $f_{i}$ is the support $\mathcal{A}_{i}$ for each $i$. (Recall that the Newton polytope is the convex hull of the corresponding lattice points.) Using the lexicographic order, we can write the elements of $\mathcal{A}_{i}$ in a unique way as $m_{i 0}, m_{i 1}, \ldots, m_{i j_{i}}$ such that $m_{i 0} \prec m_{i 1} \prec \cdots \prec m_{i j_{i}}$. Then we can write the polynomial equations as

$$
f_{1}\left(x_{1}, \ldots, x_{n}\right)=a_{j_{1}} m_{1 j_{1}}+\cdots+a_{1} m_{11}+a_{0} m_{10}
$$

and

$$
f_{2}\left(x_{1}, \ldots, x_{n}\right)=b_{j_{2}} m_{2 j_{2}}+\cdots+b_{1} m_{11}+b_{0} m_{10} .
$$

Here $m_{1 j_{1}}$ is called the leading monomial of $f_{1}, \alpha_{j_{1}} m_{1 j_{1}}$ is called the leading term of $f_{1}$, and $\alpha_{j_{1}}$ is called the leading coefficient of $f_{1}$, and similarly for $f_{2}$.

Now that we have a definite order in which to write the monomials, we can use long division to divide one polynomial by another. This is very much like long division in arithmetic (in fact, in a sense, it's easier, since there's nothing to guess). Suppose $m_{1 j_{1}}=x_{1}^{\alpha_{1}} x_{2}^{\alpha_{2}} \cdots x_{n}^{\alpha_{n}}$ and $m_{2 j_{2}}=x_{1}^{\beta_{1}} x_{2}^{\beta_{2}} \cdots x_{n}^{\beta_{n}}$. To divide $f_{1}$ by $f_{2}$, we would write $f_{1}$ and underneath it $a_{j_{1}} b_{j_{2}}^{-1} x_{1}^{\alpha_{1}-\beta_{1}} x_{2}^{\alpha_{2}-\beta_{2}} \cdots x_{n}^{\alpha_{n}-\beta_{n}} f_{2}$. When this is not a proper polynomial because $\beta_{j}>\alpha_{j}$ for some $j$, i.e., the monomial $m_{2 j_{2}}$ does not divide $m_{1 j_{1}}$, we're already done: the quotient is 0 and the remainder is $f_{2}$ itself. Otherwise, we 
subtract this from $f_{1}$ and write a term $a_{j_{1}} b_{j_{2}}^{-1} x_{1}^{\alpha_{1}-\beta_{1}} x_{2}^{\alpha_{2}-\beta_{2}} \cdots x_{n}^{\alpha_{n}-\beta_{n}}$ in the quotient. The leading term of $f_{1}$ cancels out, and the leading monomial of the difference is strictly smaller. Then we repeat this process again on this result, adding another term to the quotient, until either we can't use the leading term of $f_{2}$ to cancel the leading term of the result, or the result is zero. Then this last difference is the remainder, and we have written down all of the quotient. The process has to terminate because the leading monomials keep getting smaller and smaller.

Suppose that the two polynomial equations $f_{1}\left(x_{1}, \ldots, x_{n}\right)=0$ and $f_{2}\left(x_{1}, \ldots, x_{n}\right)$ $=0$ hold. From these two polynomial equations we can derive some more polynomial equations which are logical consequences of them. Let $\gamma_{i}=\max \left(\alpha_{i}, \beta_{i}\right)$ for $i=1, \ldots, n$, and write $\gamma=\left(\gamma_{1}, \ldots, \gamma_{n}\right)$. Since $a_{j_{1}} \neq 0$ and $b_{j_{1}} \neq 0$, we have the following equation:

$$
a_{j_{1}}^{-1} x_{1}^{\gamma_{1}-\alpha_{1}} x_{2}^{\gamma_{2}-\alpha_{2}} \cdots x_{n}^{\gamma_{n}-\alpha_{n}} f_{1}-b_{j_{2}}^{-1} x_{1}^{\gamma_{1}-\beta_{1}} x_{2}^{\gamma_{2}-\beta_{2}} \cdots x_{n}^{\gamma_{n}-\beta_{n}} f_{2}=0 .
$$

Alternatively, we could use instead the equation

$$
b_{j_{2}} x_{1}^{\gamma_{1}-\alpha_{1}} x_{2}^{\gamma_{2}-\alpha_{2}} \cdots x_{n}^{\gamma_{n}-\alpha_{n}} f_{1}-a_{j_{1}} x_{1}^{\gamma_{1}-\beta_{1}} x_{2}^{\gamma_{2}-\beta_{2}} \cdots x_{n}^{\gamma_{n}-\beta_{n}} f_{2}=0
$$

which does not require that $a_{j_{1}} \neq 0$ or $b_{j_{2}} \neq 0$. We have chosen the polynomials with which to multiply $f_{1}$ and $f_{2}$ in order to cancel the leading terms of $f_{1}$ and $f_{2}$. This polynomial is called the $S$-polynomial $S\left(f_{1}, f_{2}\right)$ of $f_{1}$ and $f_{2}$. Clearly $S\left(f_{1}, f_{2}\right)\left(x_{1}, \ldots, x_{n}\right)=0$ also.

Now we can divide $S\left(f_{1}, f_{2}\right)$ by $f_{1}$, getting an equation $S\left(f_{1}, f_{2}\right)=q_{1} f_{1}+r_{1}$ for polynomials $q_{1}$ and $r_{1}$, and then divide $r_{1}$ by $f_{2}$, getting $r_{1}=q_{2} f_{2}+r_{2}$ for polynomials $q_{2}$ and $f_{2}$. (It is unfortunately the case that the final remainder $r_{2}$ depends on the order in which we divided by $f_{1}$ and $f_{2}$.) We have that $r_{2}=S\left(f_{1}, f_{2}\right)-q_{1} f_{1}-q_{2} f_{2}$, so in particular, $r_{2}\left(x_{1}, \ldots, x_{n}\right)=0$. Thus, if $r_{2}$ is nonzero, we have a logical consequence of our polynomial equations, and we can throw it into our polynomial system. Our polynomial system is now $\left\{f_{1}, f_{2}, r_{2}\right\}$.

Now if we repeat the process, taking $S$-polynomials of pairs of polynomials in our new system and dividing each $S$-polynomial by all the polynomials in our new system, we may find more polynomial equations to throw into the system. It is a fact from commutative algebra that this process, called Buchberger's algorithm, will always terminate (i.e., finally all the remainders will be zero), and the (finite) system we have at the end is called a Gröbner basis.

Gröbner bases have many nice properties, but what will be important to us for solving polynomial systems is elimination theory. If we compute a Gröbner basis of a polynomial system in the lexicographic order with $x_{n} \preceq x_{n-1} \preceq \cdots \preceq x_{1}$, then those elements of the Gröbner basis involving only $x_{n}$ will tell us exactly the polynomial equations in $x_{n}$ alone which are logical consequences of the polynomial system. We can find the roots (if we prefer, only the real roots) of a polynomial equation in one variable, which gives us the possible values of $x_{n}$. Those elements of the Gröbner basis involving only $x_{n-1}$ and $x_{n}$ will tell us exactly the polynomial equations in $x_{n-1}$ and $x_{n}$ which are the logical consequences of the polynomial system. We can substitute in the 
possible values of $x_{n}$ we got before, to get the possible alternative polynomial equations that $x_{n-1}$ alone could satisfy. Thus we can get the possible values of $\left(x_{n-1}, x_{n}\right)$. Continuing in this way, we can get all the possible values of $\left(x_{1}, \ldots, x_{n}\right)$. If the system is positive-dimensional, we won't be able to do this $n$ times. But the generic finiteness theorem of Harsanyi (1973) tells us that a generic game has a finite number of Nash equilibria, i.e., the associated polynomial systems are zero-dimensional.

Let's use the software package Singular (Greuel et al. 2001) to compute the Gröbner basis of a game of 3 players with 2 pure strategies each.

$\sim$ Singular

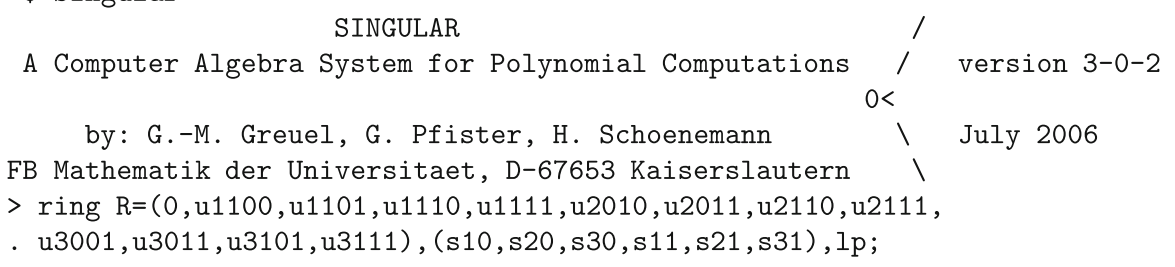

Here the second parenthesized expression gives the unknowns. As before we write $\mathrm{s} 10$ for $\sigma_{10}$ and so forth. In the first parenthesized expression, the first element denotes the characteristic of the ring. To compute over the rational numbers $\mathbb{Q}$ we set the characteristic to 0 . The rest of the elements denote parameters. In this case, the parameter ulijk denotes $u_{1}\left(s_{1 i}, s_{2 j}, s_{3 k}\right)-u_{1}\left(s_{10}, s_{2 j}, s_{3 k}\right)$, the parameter $u 2 i j k$ denotes $u_{2}\left(s_{1 i}, s_{2 j}, s_{3 k}\right)-u_{2}\left(s_{1 i}, s_{20}, s_{3 k}\right)$, and so forth. Finally, Ip means to use the lexicographic order.

Next we specify our polynomial system:

$>$ poly g1=u1100*s20*s30+u1101*s20*s31+u1110*s21*s30+u1111*s21*s31;

$>$ poly g2 $=u 2010 * \mathrm{~s} 10 * \mathrm{~s} 30+\mathrm{u} 2011 * \mathrm{~s} 10 * \mathrm{~s} 31+\mathrm{u} 2110 * \mathrm{~s} 11 * \mathrm{~s} 30+\mathrm{u} 2111 * \mathrm{~s} 11 * \mathrm{~s} 31$;

$>$ poly g3=u3001*s10*s20+u3011*s10*s21+u3101*s11*s20+u3111*s11*s21;

$>$ poly $\mathrm{g} 4=\mathrm{s} 10+\mathrm{s} 11-1$;

$>$ poly g5=s20+s21-1;

$>$ poly g6=s30+s31-1;

Finally we ask Singular to compute a Gröbner basis:

$>$ ideal $\mathrm{G}=\mathrm{g} 1, \mathrm{~g} 2, \mathrm{~g} 3, \mathrm{~g} 4, \mathrm{~g} 5, \mathrm{~g} 6$;

$>\mathrm{G}=\operatorname{groebner}(\mathrm{G})$;

We ask Singular to display the Gröbner basis it computed:

$>\mathrm{G}$;

$\mathrm{G}[1]=\mathrm{s} 30+\mathrm{s} 31-1$

$\mathrm{G}[2]=\mathrm{s} 20+\mathrm{s} 21-1$

$\mathrm{G}[3]=\mathrm{s} 10+\mathrm{s} 11-1$

$\mathrm{G}[4]=(\mathrm{u} 1100 * u 2011 * u 3101-u 1100 * u 2011 * u 3111-u 1100 * u 2111 * u 3001+$ $u 1100 * u 2111 * u 3011-u 1101 * u 2010 * u 3101+u 1101 * u 2010 * u 3111+$ $u 1101 * u 2110 * u 3001-u 1101 * u 2110 * u 3011-u 1110 * u 2011 * u 3101+$ $u 1110 * u 2011 * u 3111+u 1110 * u 2111 * u 3001-u 1110 * u 2111 * u 3011+$ $u 1111 * u 2010 * u 3101-u 1111 * u 2010 * u 3111-u 1111 * u 2110 * u 3001+$ $\mathrm{u} 1111 * u 2110 * u 3011) * \mathrm{~s} 21+$ (u1100*u2010*u3111-u1100*u2011*u3111-u1100*u2110*u3011+ 
$u 1100 * u 2111 * u 3011-u 1101 * u 2010 * u 3111+u 1101 * u 2011 * u 3111+$ u1101*u2110*u3011-u1101*u2111*u3011-u1110*u2010*u3101+ u1110*u2011*u3101+u1110*u2110*u3001-u1110*u2111*u3001+ u1111*u2010*u3101-u1111*u2011*u3101-u1111*u2110*u3001+ $\mathrm{u} 1111 * \mathrm{u} 2111 * \mathrm{u} 3001) * \mathrm{~s} 31+$

$(-u 1100 * u 2010 * u 3111-u 1100 * u 2011 * u 3101+u 1100 * u 2011 * u 3111+$ $u 1100 * u 2110 * u 3011+u 1100 * u 2111 * u 3001-u 1100 * u 2111 * u 3011+$ $u 1101 * u 2010 * u 3101-u 1101 * u 2110 * u 3001+u 1110 * u 2010 * u 3101-$ u1110*u2110*u3001-u1111*u2010*u3101+u1111*u2110*u3001)

$\mathrm{G}[5]=(-\mathrm{u} 1100 * u 2011 * u 3011+u 1100 * u 2011 * u 3111+u 1100 * u 2111 * u 3011-$ $u 1100 * u 2111 * u 3111+u 1101 * u 2010 * u 3011-u 1101 * u 2010 * u 3111-$ $u 1101 * u 2110 * u 3011+u 1101 * u 2110 * u 3111+u 1110 * u 2011 * u 3001-$ $u 1110 * u 2011 * u 3101-u 1110 * u 2111 * u 3001+u 1110 * u 2111 * u 3101-$ u1111*u2010*u3001+u1111*u2010*u3101+u1111*u2110*u3001$\mathrm{u} 1111 * \mathrm{u} 2110 * \mathrm{u} 3101) * \mathrm{~s} 11+$

$(-u 1100 * u 2010 * u 3001+u 1100 * u 2010 * u 3011+u 1100 * u 2011 * u 3001-$ $\mathrm{u} 1100 * u 2011 * u 3011+u 1100 * u 2110 * u 3001-u 1100 * u 2110 * u 3011-$ $\mathrm{u} 1100 * u 2111 * u 3001+u 1100 * u 2111 * u 3011+u 1101 * u 2010 * u 3001-$ $u 1101 * u 2010 * u 3011-u 1101 * u 2011 * u 3001+u 1101 * u 2011 * u 3011-$ $u 1101 * u 2110 * u 3001+u 1101 * u 2110 * u 3011+u 1101 * u 2111 * u 3001-$ $u 1101 * u 2111 * u 3011+u 1110 * u 2010 * u 3001-u 1110 * u 2010 * u 3011-$ $u 1110 * u 2011 * u 3001+u 1110 * u 2011 * u 3011-u 1110 * u 2110 * u 3001+$ $u 1110 * u 2110 * u 3011+u 1110 * u 2111 * u 3001-u 1110 * u 2111 * u 3011-$ u1111*u2010*u3001+u1111*u2010*u3011+u1111*u2011*u3001$u 1111 * u 2011 * u 3011+u 1111 * u 2110 * u 3001-u 1111 * u 2110 * u 3011-$ $u 1111 * u 2111 * u 3001+u 1111 * u 2111 * u 3011) * s 21 * s 31+$

(u1100*u2010*u3001-u1100*u2010*u3011-u1100*u2011*u3001+ u1100*u2011*u3011-u1100*u2110*u3001+u1100*u2110*u3011+ u1100*u2111*u3001-u1100*u2111*u3011-u1110*u2010*u3001+ $u 1110 * u 2010 * u 3011+u 1110 * u 2011 * u 3001-u 1110 * u 2011 * u 3011+$ u1110*u2110*u3001-u1110*u2110*u3011-u1110*u2111*u3001+ $\mathrm{u} 1110 * \mathrm{u} 2111 * \mathrm{u} 3011) * \mathrm{~s} 21+$

(u1100*u2010*u3001-u1100*u2010*u3011+u1100*u2010*u3111$u 1100 * u 2011 * u 3001+u 1100 * u 2011 * u 3011-u 1100 * u 2011 * u 3111-$ $u 1100 * u 2110 * u 3001+u 1100 * u 2111 * u 3001-u 1101 * u 2010 * u 3001+$ u1101*u2010*u3011-u1101*u2010*u3111+u1101*u2011*u3001u1101*u2011*u3011+u1101*u2011*u3111+u1101*u2110*u3001u1101*u2111*u3001-u1110*u2010*u3101+u1110*u2011*u3101+ $u 1110 * u 2110 * u 3001-u 1110 * u 2111 * u 3001+u 1111 * u 2010 * u 3101-$ $u 1111 * u 2011 * u 3101-u 1111 * u 2110 * u 3001+u 1111 * u 2111 * u 3001$ )

* $\mathrm{s} 31+$

$(-u 1100 * u 2010 * u 3001+u 1100 * u 2010 * u 3011-u 1100 * u 2010 * u 3111+$ $u 1100 * u 2011 * u 3001+u 1100 * u 2110 * u 3001-u 1100 * u 2111 * u 3001-$ $u 1101 * u 2010 * u 3011+u 1101 * u 2010 * u 3111+u 1110 * u 2010 * u 3101-$ $u 1110 * u 2011 * u 3001-u 1110 * u 2110 * u 3001+u 1110 * u 2111 * u 3001+$ u1111*u2010*u3001-u1111*u2010*u3101)

$\mathrm{G}[6]=(-\mathrm{u} 1100 * u 2010 * u 3111+u 1100 * u 2011 * u 3111+$ 
$u 1100 * u 2110 * u 3011-u 1100 * u 2111 * u 3011+u 1101 * u 2010 * u 3111-$ u1101*u2011*u3111-u1101*u2110*u3011+u1101*u2111*u3011+ u1110*u2010*u3101-u1110*u2011*u3101-u1110*u2110*u3001+ u1110*u2111*u3001-u1111*u2010*u3101+u1111*u2011*u3101+ $\mathrm{u} 1111 * \mathrm{u} 2110 * \mathrm{u} 3001-\mathrm{u} 1111 * \mathrm{u} 2111 * \mathrm{u} 3001) * \mathrm{~s} 31^{\wedge} 2+$

$(2 * u 1100 * u 2010 * u 3111-u 1100 * u 2011 * u 3111-$

$2 * u 1100 * u 2110 * u 3011+u 1100 * u 2111 * u 3011-u 1101 * u 2010 * u 3111+$ u1101*u2110*u3011-2*u1110*u2010*u3101+u1110*u2011*u3101+ 2 *u1110*u2110*u3001-u1110*u2111*u3001+u1111*u2010*u3101$\mathrm{u} 1111 * \mathrm{u} 2110 * \mathrm{u} 3001) * \mathrm{~s} 31+$

$(-u 1100 * u 2010 * u 3111+u 1100 * u 2110 * u 3011+u 1110 * u 2010 * u 3101-$ $u 1110 * u 2110 * u 3001)$

(We have reformatted the output.) The Gröbner basis has 6 elements. The first three elements tell us how to find s10 in terms of s11, s2 0 in terms of s21, and s 30 in terms of s31. The last element G [6] is a quadratic polynomial in s31 alone. We can solve this equation to find the possible values of s31. The fourth element G [4] tells us how to obtain s21 once we have s31, and the fifth element G [5] tells us how to obtain s11 once we have s31 and s 21 .

Any particular $2 \times 2 \times 2$ game is specified by particular values of the parameters, so we can just substitute them in and solve the resulting system. Having the Gröbner basis gives us important information about how the geometry of the solution set varies with the parameters. For instance, if the coefficient of $s 31^{2}$ in G [6] vanishes, then this polynomial only has degree 1 and hence only one real root. We can consider the discriminant of the quadratic equation G [6]. Writing $u_{s}^{i}=u_{i}\left(s_{i j}, s_{-i}\right)$, the discriminant becomes:

$$
\begin{aligned}
& \left(2 u_{100}^{1} u_{010}^{2} u_{111}^{3}-u_{100}^{1} u_{011}^{2} u_{111}^{3}-2 u_{100}^{1} u_{110}^{2} u_{011}^{3}+u_{100}^{1} u_{111}^{2} u_{011}^{3}-u_{101}^{1} u_{010}^{2} u_{111}^{3}\right. \\
& +u_{101}^{1} u_{110}^{2} u_{011}^{3}-2 u_{110}^{1} u_{010}^{2} u_{101}^{3}+u_{110}^{1} u_{011}^{2} u_{101}^{3}+2 u_{110}^{1} u_{110}^{2} u_{001}^{3}-u_{110}^{1} u_{111}^{2} u_{001}^{3} \\
& \left.+u_{111}^{1} u_{010}^{2} u_{101}^{3}-u_{111}^{1} u_{110}^{2} u_{001}^{3}\right)^{2}-4\left(-u_{100}^{1} u_{010}^{2} u_{111}^{3}+u_{100}^{1} u_{011}^{2} u_{111}^{3}\right. \\
& +u_{100}^{1} u_{110}^{2} u_{011}^{3}-u_{100}^{1} u_{111}^{2} u_{011}^{3}+u_{101}^{1} u_{010}^{2} u_{111}^{3}-u_{101}^{1} u_{011}^{2} u_{111}^{3}-u_{101}^{1} u_{110}^{2} u_{011}^{3} \\
& +u_{101}^{1} u_{111}^{2} u_{011}^{3}+u_{110}^{1} u_{010}^{2} u_{101}^{3}-u_{110}^{1} u_{011}^{2} u_{101}^{3}-u_{110}^{1} u_{110}^{2} u_{001}^{3}+u_{110}^{1} u_{111}^{2} u_{001}^{3} \\
& \left.-u_{111}^{1} u_{010}^{2} u_{101}^{3}+u_{111}^{1} u_{011}^{2} u_{101}^{3}+u_{111}^{1} u_{110}^{2} u_{001}^{3}-u_{111}^{1} u_{111}^{2} u_{001}^{3}\right) \\
& \times\left(-u_{100}^{1} u_{010}^{2} u_{111}^{3}+u_{100}^{1} u_{110}^{2} u_{011}^{3}+u_{110}^{1} u_{010}^{2} u_{101}^{3}-u_{110}^{1} u_{110}^{2} u_{001}^{3}\right) .
\end{aligned}
$$

The set of payoff functions where the discriminant is zero is a real algebraic variety in the space of $2 \times 2 \times 2$ games, the discriminant variety. It partitions the space of $2 \times 2 \times 2$ games into a region where the discriminant is positive, in which case the polynomial system has two real roots, and a region where the discriminant is negative, in which case the polynomial system has no real roots.

The reader was already familiar with the discriminant of the quadratic formula, but the same phenomenon will happen with equations of higher degrees in more variables (Gelfand et al. 1994). We saw in our example that only a single polynomial of the 
Gröbner basis was nonlinear, and this is true in general for generic games in normal form, by Theorem 9 of Herings and Peeters (2005). For higher-degree polynomial systems there could be several discriminant varieties, with various implications about the geometry of the solution set. The SALSA team of INRIA Rocquencourt and LIP6 in France has produced a Maple package DV which will analyze the discriminant varieties for a parametric polynomial system Lazard and Rouillier (2007), and Montes (2002) has independently produced another such Maple package DisPGB. (These analyze the implications of the discriminantal equations being zero or nonzero for the complex solutions of the polynomial system. In the example above, we considered in addition the implications of the discriminant being positive or negative on the real solutions.)

Specifically, Gabriela Jeronimo, Daniel Perrucci, and Juan Sabia have recently explained how to obtain a parametric representation of the totally mixed Nash equilibria. (As we know, this means we can get a parametric representation of all the Nash equilibria by considering various possible supports.) They give polynomial-time algorithms for describing the set of totally mixed Nash equilibria, using multihomogeneous resultants. The resultant of a polynomial system is a polynomial equation in the coefficients of the system which must hold in order for the system to have a root. Resultants are a key tool in the solution of polynomial systems. The parametric equations characterize the geometry of the space of games and provide an effective method for finding all the roots. We look forward eagerly to the implementation of these algorithms in a software package, which we hope will lead to many new insights in game theory.

\section{Finding all Nash equilibria in Gambit}

The Gambit software package incorporates a variety of tools for finding Nash equilibria and studying other properties of games. We briefly discuss here the method used by Gambit to find all Nash equilibria prior to the release of 30 January 2007. (This release, current as of this writing, includes an experimental option to use PHC to find all Nash equilibria.)

Gambit solves the polynomial systems defining the Nash equilibria by subdividing the product of simplices (where each simplex is the subset of $\mathbb{R}^{d_{i}}$ defined by $\sigma_{i 1}+\cdots+\sigma_{i d_{i}}=1, \sigma_{i j} \geq 0$ for all $j$ ) into small boxes and looking at the Taylor series of each polynomial in the system. We can, for instance, evaluate the $D$ polynomials $\left(f_{1}, \ldots, f_{D}\right)$ at one corner $\sigma$ of such a box, giving a vector $\left(y_{1}, \ldots, y_{D}\right)$. Then any point in the box is no further than the diagonally opposite corner, and we can plug this distance into the Taylor series of the polynomials about the corner to determine a bound on how far the image of any other point $\sigma^{\prime}$ of the box can lie from $\left(y_{1}, \ldots, y_{D}\right)$. If this bound is less than $\left\|\left(y_{1}, \ldots, y_{D}\right)\right\|_{2}$, then no point in the box can be a root of the polynomial system. If it is not, then Gambit looks for a root in the box using Newton's method. If such a root is found, then Gambit tries to see whether it can determine that no other roots may exist within the box, again using the Taylor series of the polynomials. If it can, then it is done with this box, but if not, then it subdivides the box into $2^{D}$ smaller boxes and looks at those.

This method has the advantage that it will only find real roots which satisfy the nonnegativity constraints. However, it does not scale well with higher dimensions. Using 
polyhedral homotopy continuation with factorizable starting systems, finding all Nash equilibria of games of much larger formats should become practical. However, it may be useful to use a similar Taylor series technique on the augmented system (the one including the variable $t$ ) along the way to each root of the target system, to see whether we can derive a bound ensuring that the target root will not be real and nonnegative (i.e., a Nash equilibrium). In that case we can abandon this particular path. Here we are just travelling along a 1 -dimensional interval $[0,1]$, so the problem of having to subdivide into an exponential number $2^{D}$ of smaller boxes does not arise.

Gambit also provides an option to compute Nash equilibria via heuristic search on the supports of the game (Porter et al. 2008), through an implementation contributed by Litao Wei. Heuristic search is complementary to using polynomial algebra to find Nash equilibria. It can tell us which of the many possible supports to look at first. The choice of support tells us which polynomial systems to solve (possibly none, if we find a pure Nash equilibrium on the support and are satisfied with not looking any further).

\section{Conclusion}

In this paper, we have described the polynomial systems which characterize the Nash equilibria of a game. We have explained how to construct and solve a factorizable start system and then use polyhedral homotopy continuation to solve games of a given format. We have also explained how to use Gröbner bases to gain insight into how the geometry of the solution set of these polynomial systems varies over the space of games of a particular format. Finally, we have reviewed the current use of Gambit for finding all Nash equilibria of a game. We suggest that Gambit may be able to find all Nash equilibria of games of larger formats than is currently possible, by incorporating polyhedral homotopy continuation from factorizable nondegenerate start systems. Alternatively, or in addition, Gambit may implement the algorithm of Jeronimo, Perrucci, and Sabia to find all Nash equilibria of a game. We sincerely hope that the possibility of analyzing larger games will enable game theorists to make more realistic models of strategic interaction.

\section{Appendix}

In this appendix, we eliminate the complementary slack variables from the system $(*)$. We carry out steps similar to the steps carried out by a Gröbner basis algorithm, but in this case we only need elementary methods. We find that for a generic game, at each solution either for every player all but one of the complementary slack variables are positive, corresponding to a pure strict Nash equilibrium, or for at least two players at least two of them are zero.

Fix a player $i \in I$, a pure strategy $s_{i j} \in S_{i}$ of player $i$, and a pure strategy $s_{k l_{k}} \in S_{k}$ for every other player $k \in I-\{i\}$, giving a pure $(-i)$-strategy profile $s_{-i} \in S_{-i}$. Then the system $(*)$ implies that

$$
u_{i}\left(s_{i j}, \sigma_{-i}\right)+v_{i j}=u_{i}\left(s_{i 0}, \sigma_{-i}\right)+v_{i 0}
$$


Multiplying through by

$$
\prod_{k \in I-\{i\}} \prod_{\substack{m_{k}=0 \\ m_{k} \neq l_{k}}}^{d_{k}} v_{k m_{k}}
$$

which we denote by $p\left(s_{-i}\right)$, and using that $\sigma_{k m_{k}} v_{k m_{k}}=0$, we find that

$$
\begin{aligned}
& \left(u_{i}\left(s_{i j}, s_{-i}\right) \prod_{k \in I-\{i\}} \sigma_{k l_{k}}+v_{i j}\right) \prod_{k \in I-\{i\}} \prod_{\substack{m_{k}=0 \\
m_{k} \neq l_{k}}}^{d_{k}} v_{k m_{k}} \\
& =\left(u_{i}\left(s_{i 0}, s_{-i}\right) \prod_{k \in I-\{i\}} \sigma_{k l_{k}}+v_{i 0}\right) \prod_{k \in I-\{i\}} \prod_{\substack{m_{k}=0 \\
m_{k} \neq l_{k}}}^{d_{k}} v_{k m_{k}} .
\end{aligned}
$$

Every other term in $u_{i}\left(s_{i j}, \sigma_{-i}\right)$ is killed by one or more of the $v_{k m_{k}}$ 's, and similarly for $u_{i}\left(s_{i 0}, \sigma_{-i}\right)$. Next, we substitute $1-\sum_{\substack{m_{k}=0 \\ m_{k} \neq l_{k}}}^{d_{k}} \sigma_{k m_{k}}$ for $\sigma_{k l_{k}}$ in each factor of the product $\prod_{k \in I-\{i\}} \sigma_{k l_{k}}$, and note that every nonconstant term in the resulting polynomial is also killed by one or more of the $v_{k m_{k}}$ 's. Finally, we have

$$
\left(v_{i j}-v_{i 0}+u_{i}\left(s_{i j}, s_{-i}\right)-u_{i}\left(s_{i 0}, s_{-i}\right)\right) \prod_{k \in I-\{i\}} \prod_{\substack{m_{k}=0 \\ m_{k} \neq l_{k}}}^{d_{k}} v_{k m_{k}}=0,
$$

an equation involving only the complementary slack variables. Clearly, for this equation to hold, either $v_{i j}-v_{i 0}+u_{i}\left(s_{i j}, s_{-i}\right)-u_{i}\left(s_{i 0}, s_{-i}\right)$ or one of the $v_{k m_{k}}$ 's must vanish.

In this way we obtain a system of equations in the $v_{i j}$ 's, which we denote by $(\mathbf{V})$. Denote by $\left(\mathbf{V}_{i}\right)$ the subsystem corresponding to fixing player $i$ above, and by $\left(\mathbf{V}_{i, s_{-i}}\right)$ the subsystem corresponding to fixing player $i$ and the pure $(-i)$-strategy profile $s_{-i}$.

If $p\left(s_{-i}\right) \neq 0$ for some $s_{-i} \in S_{-i}$, then the system of equations $\left(\mathbf{V}_{i, s_{-i}}\right)$ reduces to $v_{i j}=v_{i 0}+u_{i}\left(s_{i 0}, s_{-i}\right)-u_{i}\left(s_{i j}, s_{-i}\right)$ for each $j=1, \ldots, d_{i}$. The solutions to this system along with the nonnegativity constraints $v_{i j} \geq 0$ lie along a ray parametrized by $v_{i 0}$, with

$$
v_{i 0} \geq \max _{j=0}^{d_{i}}\left(u_{i}\left(s_{i j}, s_{-i}\right)-u_{i}\left(s_{i 0}, s_{-i}\right)\right)
$$

Only for those $j=0, \ldots, d_{i}$ at which $u_{i}\left(s_{i j}, s_{-i}\right)-u_{i}\left(s_{i 0}, s_{-i}\right)$ attains its maximum can $v_{i j}$ ever vanish; the rest of the $v_{i j}$ 's must be positive. But since $\sum_{j=0}^{d_{i}} \sigma_{i j}=1$ with $\sigma_{i j} \geq 0$, at least one $\sigma_{i j}$ must be positive, and thus since $\sigma_{i j} v_{i j}=0$ for all $j$, at least one $v_{i j}$ must vanish. Therefore we have a unique solution, the point with $v_{i 0}=\max _{j=0}^{d_{i}}\left(u_{i}\left(s_{i j}, s_{-i}\right)-u_{i}\left(s_{i 0}, s_{-i}\right)\right)$. Let us denote this point by $q\left(s_{-i}\right) \in \mathbb{R}_{\geq 0}^{d_{i}+1}$. 
For generic games, the point $q\left(s_{-i}\right)$ corresponding to $s_{-i}$ will be different from the point $q\left(s_{-i}^{\prime}\right)$ corresponding to $s_{-i}^{\prime}$ for any other $s_{-i}^{\prime} \in S_{-i}$. By this we mean that for $q\left(s_{-i}\right)$ to equal $q\left(s_{-i}^{\prime}\right)$ imposes an extra condition on the payoff function, which is only satisfied on a semialgebraic subvariety of the space of games of lower dimension. For a fixed game format, the set of all payoff functions is a finite-dimensional real vector space whose coordinates are $u_{i}(s)$ for all players $i$ and pure strategy profiles $s$. This is the space of games of that format. The condition that $q\left(s_{-i}\right)$ equals $q\left(s_{-i}^{\prime}\right)$ is equivalent to one of a set of alternatives, each of which is an equation and a set of inequalities. The equation defines a hyperplane in the space of games, and so is of lower dimension. So generically, $q\left(s_{-i}\right)$ is different from $q\left(s_{-i}^{\prime}\right)$. This also implies that $q\left(s_{-i}\right) \neq q\left(s_{-i}^{\prime}\right)$ almost everywhere, and that $q\left(s_{-i}\right) \neq q\left(s_{-i}^{\prime}\right)$ on a dense open subset of the space of games.

Thus for generic games, the system $\left(\mathbf{V}_{i}\right)$ reduces to the following $\left|S_{-i}\right|+1$ alternatives: either

$$
p\left(s_{-i}\right)=0 \text { for all } s_{-i} \in S_{-i},
$$

or for some $s_{-i}^{*} \in S_{-i}$, we have the system $\left(\mathbf{V}_{i}^{s_{-i}^{*}}\right)$ :

$$
p\left(s_{-i}\right)=0 \text { for all } s_{-i} \in S_{-i}-\left\{s_{-i}^{*}\right\}
$$

and

$$
\left(v_{i 0}, \ldots, v_{i d_{i}}\right)=q\left(s_{-i}^{*}\right) \text {. }
$$

For generic games, exactly one of the $v_{i j}$ 's will vanish at the point $q\left(s_{-i}^{*}\right)$.

We now introduce some notation from logic. The symbol $\wedge$ means "and" and the symbol $\vee$ means "or" (in the Boolean sense). Suppose $\varphi_{0}$ and $\varphi_{1}$ are Boolean expressions. Then the expression $\varphi_{0} \wedge \varphi_{1}$, meaning " $\varphi_{0}$ and $\varphi_{1}$ ", is the conjunction of $\varphi_{0}$ and $\varphi_{1}$. The expression $\varphi_{0} \vee \varphi_{1}$, meaning " $\varphi_{0}$ or $\varphi_{1}$ ", is the disjunction of $\varphi_{0}$ and $\varphi_{1}$. Suppose $\varphi_{0}, \ldots, \varphi_{n}$ are Boolean expressions. Then $\bigwedge_{i=0}^{n} \varphi_{i}$ denotes the conjunction of $\varphi_{0}, \ldots, \varphi_{n}$, and $\bigvee_{i=0}^{n} \varphi_{i}$ denotes the disjunction of $\varphi_{0}, \ldots, \varphi_{n}$. The distributive law holds for conjunction and disjunction just as it holds for multiplication and addition.

Lemma 1 The solutions of the system $\left(\mathbf{V}_{i}^{p}\right)$ are given by

$$
\bigvee_{\substack{k=1 \\ k \neq i}}^{N} \bigvee_{j=0}^{d_{k}} \bigvee_{\substack{l=1 \\ l>j}}^{d_{k}}\left(v_{k j}=0\right) \wedge\left(v_{k l}=0\right)
$$

Proof We proceed by induction on the number $N$ of players. First suppose $N=2$. Without loss of generality, fix $i=2$. Then each $s_{-i}$ is $s_{1 j}$ for some $j$. The equation $\left(\mathbf{V}_{2, s_{1 j}}^{p}\right)$ is $p\left(s_{1 j}\right)=\prod_{\substack{l=0 \\ l \neq j}}^{d_{1}} v_{1 l}=0$, which holds if and only if $\bigvee_{\substack{l=0 \\ l \neq j}}^{d_{1}}\left(v_{1 l}=0\right)$. So the system $\left(\mathbf{V}_{2}^{p}\right)$ holds if and only if $\bigwedge_{j=0}^{d_{1}} \bigvee_{\substack{l=0 \\ l \neq j}}^{d_{1}}\left(v_{1 l}=0\right)$. We show by induction on 
$d_{1}$ that this expression is equal to $\bigvee_{j=0}^{d_{1}} \bigvee_{\substack{l=1 \\ l>j}}^{d_{1}}\left(v_{1 j}=0\right) \wedge\left(v_{1 l}=0\right)$ which equals $\bigvee_{\substack{k=1 \\ k \neq 2}}^{2} \bigvee_{j=0}^{d_{k}} \bigvee_{\substack{l=1 \\ l>j}}^{d_{k}}\left(v_{k j}=0\right) \wedge\left(v_{k l}=0\right)$. For $d_{1}=1$

$$
\begin{aligned}
\bigwedge_{j=0}^{1} \bigvee_{\substack{l=0 \\
l \neq j}}^{1}\left(v_{1 l}=0\right) & =\bigwedge_{j=0}^{1}\left(v_{1(1-j)}=0\right) \\
& =\left(v_{11}=0\right) \wedge\left(v_{10}=0\right) \\
& =\bigvee_{j=0}^{1} \bigvee_{\substack{l=1 \\
l>j}}^{1}\left(v_{1 j}=0\right) \wedge\left(v_{1 l}=0\right)
\end{aligned}
$$

Now assume that $\bigwedge_{j=1}^{d_{1}} \bigvee_{\substack{l=1 \\ l \neq j}}^{d_{1}}\left(v_{1 l}=0\right)=\bigvee_{j=1}^{d_{1}} \bigvee_{\substack{l=2 \\ l>j}}^{d_{1}}\left(v_{1 j}=0\right) \wedge\left(v_{1 l}=0\right)$. Then

$$
\begin{aligned}
& \bigwedge_{j=0}^{d_{1}} \bigvee_{\substack{l=0 \\
l \neq j}}^{d_{1}}\left(v_{1 l}=0\right) \\
& =\left(\bigvee_{l=1}^{d_{1}}\left(v_{1 l}=0\right)\right) \wedge \bigwedge_{j=1}^{d_{1}} \bigvee_{\substack{l=0 \\
l \neq j}}^{d_{1}}\left(v_{1 l}=0\right) \\
& =\left(\bigvee_{l=1}^{d_{1}}\left(v_{1 l}=0\right)\right) \wedge \bigwedge_{j=1}^{d_{1}}\left(\left(v_{10}=0\right) \vee \bigvee_{\substack{l=1 \\
l \neq j}}^{d_{1}}\left(v_{1 l}=0\right)\right) \\
& =\left(\bigvee_{l=1}^{d_{1}}\left(v_{1 l}=0\right)\right) \wedge\left(\left(v_{10}=0\right) \vee \bigwedge_{\substack{d_{1} \\
l \neq 1 \\
l \neq j}}^{d_{1}}\left(v_{1 l}=0\right)\right) \\
& =\left(\bigvee_{l=1}^{d_{1}}\left(v_{1 l}=0\right)\right) \wedge\left(\left(v_{10}=0\right) \vee \bigvee_{j=1}^{d_{1}} \bigvee_{\substack{l=2 \\
l>j}}^{d_{1}}\left(v_{1 j}=0\right) \wedge\left(v_{1 l}=0\right)\right) \\
& =\left(\bigvee_{l=1}^{d_{1}}\left(v_{10}=0\right) \wedge\left(v_{1 l}=0\right)\right) \\
& \times \vee\left(( \bigvee _ { l = 1 } ^ { d _ { 1 } } ( v _ { 1 l } = 0 ) ) \wedge \bigvee _ { j = 1 } ^ { d _ { 1 } } \left(( v _ { 1 j } = 0 ) \wedge \left(\bigvee_{\substack{d_{1} \\
l>j}}^{\left.\left.\left.d_{l}\left(v_{1 l}=0\right)\right)\right)\right)}\right.\right.\right. \\
& =\left(\bigvee_{j=0}^{0} \bigvee_{\substack{l=1 \\
l>j}}^{d_{1}}\left(v_{1 j}=0\right) \wedge\left(v_{1 l}=0\right)\right)
\end{aligned}
$$




$$
\begin{aligned}
& \times \vee\left(\left(\bigvee_{j=1}^{d_{1}}\left(v_{1 j}=0\right)\right) \wedge \bigvee_{j=1}^{d_{1}}\left(\left(v_{1 j}=0\right) \wedge\left(\bigvee_{\substack{l_{1} \\
l>j}}^{d_{1}}\left(v_{1 l}=0\right)\right)\right)\right) \\
= & \left(\bigvee_{j=0}^{0} \bigvee_{\substack{l=1 \\
l>j}}^{d_{1}}\left(v_{1 j}=0\right) \wedge\left(v_{1 l}=0\right)\right) \vee \bigvee_{j=1}^{d_{1}}\left(\left(v_{1 j}=0\right) \wedge\left(\bigvee_{\substack{l=1 \\
l>j}}^{d_{1}}\left(v_{1 l}=0\right)\right)\right) \\
= & \bigvee_{j=0}^{d_{i} \bigvee_{\substack{l=1 \\
l>j}}^{d_{1}}\left(v_{1 j}=0\right) \wedge\left(v_{1 l}=0\right) .}
\end{aligned}
$$

Now assume the lemma holds for any number of players less than $N$. Fix $i \in I-\{N\}$. Suppose that $\bigvee_{\substack{k=1 \\ k \neq i}}^{N} \bigvee_{j=0}^{d_{k}} \bigvee_{\substack{l=1 \\ l>j}}^{d_{k}}\left(v_{k j}=0\right) \wedge\left(v_{k l}=0\right)$ holds. Fix $n \in I-\{i\}$ and $j, m \in S_{n}$ with $j \neq m$ such that $v_{n j}=0$ and $v_{n m}=0$. Consider a pure strategy $s_{k l_{k}} \in S_{k}$ for every player $k \in I-\{i\}$, giving a pure $(-i)$-strategy profile $s_{-i} \in S_{-i}$. The equation $\left(\mathbf{V}_{i, s_{-i}}^{p}\right)$ is

$$
p\left(s_{-i}\right)=\prod_{k \in I-\{i\}} \prod_{\substack{m_{k}=0 \\ m_{k} \neq l_{k}}}^{d_{k}} v_{k m_{k}}=0 .
$$

Either $l_{n}=j$, in which case the product vanishes since it includes $v_{n m}$; or $l_{n}=m$, in which case the product vanishes since it includes $v_{n j}$; or $s_{n l_{n}} \in S_{n}-\left\{s_{n j}, s_{n m}\right\}$, in which case the product vanishes since it includes both $v_{n j}$ and $v_{n m}$. Thus the equation $\left(\mathbf{V}_{i, s_{-i}}^{p}\right)$ holds. So the system $\left(\mathbf{V}_{i}^{p}\right)$ is satisfied.

Conversely, suppose the system $\left(\mathbf{V}_{i}^{p}\right)$ holds. If there exist $j, m \in S_{N}$ with $j \neq m$ such that $v_{N j}=0$ and $v_{N m}=0$, then $\bigvee_{\substack{k=1 \\ k \neq i}}^{N} \bigvee_{j=0}^{d_{k}} \bigvee_{\substack{l=1 \\ l>j}}^{d_{k}}\left(v_{k j}=0\right) \wedge\left(v_{k l}=0\right)$ holds, so assume there do not exist such $j, m \in S_{N}$. Then there exists $j \in S_{N}$ such that $v_{N m} \neq 0$ for all $s_{N m} \in S_{N}-\left\{s_{N j}\right\}$. Write $S_{-i}^{\prime}=\prod_{k \in I-\{i, N\}} S_{k}$. Consider all elements of $S_{-i}$ given by pure strategies $s_{k l_{k}} \in S_{k}$ for $k \in I-\{i, N\}$ and $s_{N l_{N}}=s_{N j} \in S_{N}$. Thus $s_{-i}$ has the form $s_{-i}=\left(s_{-i}^{\prime}, s_{N j}\right)$ for some $s_{i}^{\prime} \in S_{i}^{\prime}$. The equation $\left(\mathbf{V}_{i, s_{-i}}^{p}\right)$ is

$$
p\left(s_{-i}\right)=\prod_{k \in I-\{i\}} \prod_{\substack{m_{k}=0 \\ m_{k} \neq l_{k}}}^{d_{k}} v_{k m_{k}}=\left(\prod_{k \in I-\{i, N\}} \prod_{\substack{m_{k}=0 \\ m_{k} \neq l_{k}}}^{d_{k}} v_{k m_{k}}\right) \prod_{\substack{m=0 \\ m \neq j}}^{d_{N}} v_{N m}=0 .
$$

Since $v_{N m} \neq 0$ for all $s_{N m} \in S_{N}-\left\{s_{N j}\right\}$, this equation is equivalent to the equation $p\left(s_{-i}^{\prime}\right)=\prod_{k \in I-\{i, N\}} \prod_{\substack{d_{k} \\ m_{k}=0 \\ m_{k} \neq l_{k}}}^{d_{k m_{k}}}=0$. By the induction hypothesis, these equations imply that $\bigvee_{\substack{k=1 \\ k \neq i}}^{N-1} \bigvee_{j=0}^{d_{k}} \bigvee_{\substack{l=1 \\>>j}}^{d_{k}}\left(v_{k j}=0\right) \wedge\left(v_{k l}=0\right)$ holds. Thus $\bigvee_{\substack{k=1 \\ k \neq i}}^{N} \bigvee_{j=0}^{d_{k}} \bigvee_{\substack{l=1 \\ l>j}}^{d_{k}}$ $\left(v_{k j}=0\right) \wedge\left(v_{k l}=0\right)$ holds. 
Lemma 2 Fix $i \in I$ and fix pure strategies $s_{k l_{k}} \in S_{k}$ for each $k \in I-\{i\}$, defining $a$ $(-i)$-strategy profile $s_{-i}^{*} \in S_{-i}$. Then the solutions of the system $\left(\mathbf{V}_{i}^{s_{-i}^{*} p}\right)$ are given by

$$
\bigwedge_{\substack{k=1 \\ k \neq i}}^{N}\left(v_{k l_{k}}=0\right) \vee \bigvee_{\substack{k=1 \\ k \neq i}}^{N} \bigvee_{j=0}^{d_{k}} \bigvee_{\substack{l=1 \\ l>j}}^{d_{k}}\left(v_{k j}=0\right) \wedge\left(v_{k l}=0\right)
$$

Proof Suppose $\bigwedge_{\substack{k=1 \\ k \neq i}}^{N}\left(v_{k l_{k}}=0\right) \vee \bigvee_{\substack{k=1 \\ k \neq i}}^{N} \bigvee_{j=0}^{d_{k}} \bigvee_{\substack{d_{k}=1 \\ l>j}}^{d_{k}}\left(v_{k j}=0\right) \wedge\left(v_{k l}=0\right)$ holds. If

$$
\begin{aligned}
& \bigvee^{N} \bigvee^{d_{k}} \bigvee^{d_{k}}\left(v_{k j}=0\right) \wedge\left(v_{k l}=0\right) \\
& \begin{array}{ll}
k=1 \\
k \neq i
\end{array} \quad j=0 \begin{array}{l}
l=1 \\
l>j
\end{array}
\end{aligned}
$$

holds, then by Lemma 1 , the system $\left(\mathbf{V}_{i}^{p}\right)$ holds so a fortiori the system $\left(\mathbf{V}_{i}^{s_{-i}^{*} p}\right)$ holds. Suppose $\bigwedge_{\substack{k=1 \\ k \neq i}}^{N}\left(v_{k l_{k}}=0\right)$ holds. Let $s_{-i} \in S_{-i}-\left\{s_{-i}^{*}\right\}$ with components $s_{k n_{k}} \in S_{k}$. Then

$$
p\left(s_{-i}\right)=\prod_{\substack{k=1 \\ k \neq i}}^{N} \prod_{\substack{m_{k}=0 \\ m_{k} \neq n_{k}}}^{d_{k}} v_{k m_{k}}
$$

Since $s_{-i} \neq s_{-i}^{*}$, there is some $k \in I-\{i\}$ such that $n_{k} \neq l_{k}$. Then $v_{k l_{k}}$ appears in the product, and so $p\left(s_{-i}\right)=0$. So the system $\left(\mathbf{V}_{i}^{s_{-i}^{*} p}\right)$ holds.

Conversely, suppose $\bigwedge_{\substack{k=1 \\ k \neq i}}^{N}\left(v_{k l_{k}}=0\right) \vee \bigvee_{\substack{k=1 \\ k \neq i}}^{N} \bigvee_{j=0}^{d_{k}} \bigvee_{\substack{l=1 \\ l>j}}^{d_{k}}\left(v_{k j}=0\right) \wedge\left(v_{k l}=0\right)$ does not hold. Since $\bigwedge_{\substack{k=1 \\ k \neq i}}^{N}\left(v_{k l_{k}}=0\right)$ does not hold, there is some $k^{\prime} \in I-\{i\}$ such that $v_{k^{\prime}} l_{k^{\prime}} \neq 0$. Also since $\bigvee_{\substack{k=1 \\ k \neq i}}^{N} \bigvee_{j=0}^{d_{k}} \bigvee_{\substack{l=1 \\ l>j}}^{d_{k}}\left(v_{k j}=0\right) \wedge\left(v_{k l}=0\right)$ does not hold, for each $k \in I-\{i\}$, either $v_{k j} \neq 0$ for all $s_{k j} \in S_{k}$, or $v_{k n_{k}}=0$ for a single $s_{k n_{k}} \in S_{k}$ and $v_{k m_{k}} \neq 0$ for all $m_{k}$ with $s_{k m_{k}} \in S_{k}-\left\{s_{k n_{k}}\right\}$. In either case, there is some $n_{k}$ with $s_{k n_{k}} \in S_{k}$ such that $v_{k m_{k}} \neq 0$ for all $m_{k}$ with $s_{k m_{k}} \in S_{k}-\left\{s_{k n_{k}}\right\}$. In particular, for $k^{\prime}$ we can choose $n_{k^{\prime}} \neq l_{k^{\prime}}$, because in the former case, any $n_{k^{\prime}}$ other than $l_{k^{\prime}}$ will do, and in the latter case, the single $n_{k^{\prime}}$ with $v_{k n_{k^{\prime}}}=0$ must not be equal to $l_{k^{\prime}}$, since $v_{k^{\prime} l_{k^{\prime}}} \neq 0$. Define $s_{-i} \in S_{-i}$ with components $s_{k n_{k}} \in S_{k}$ such that $v_{k m_{k}} \neq 0$ for all $m_{k}$ with $s_{k m_{k}} \in S_{k}-\left\{s_{k n_{k}}\right\}$, for all $k \in I-\left\{i, k^{\prime}\right\}$, and $n_{k^{\prime}} \neq l_{k^{\prime}}$. Then

$$
p\left(s_{-i}\right)=\prod_{\substack{k=1 \\ k \neq i}}^{N} \prod_{\substack{m_{k}=0 \\ m_{k} \neq n_{k}}}^{d_{k}} v_{k m_{k}} \neq 0
$$


by the choice of the $n_{k}$ 's. Since $n_{k^{\prime}} \neq l_{k^{\prime}}$, we have $s_{-i} \in S_{-i}-\left\{s_{-i}^{*}\right\}$. So $\left(\mathbf{V}_{i, s_{-i}}^{s_{-i}^{*} p}\right)$ does not hold and the system $\left(\mathbf{V}_{i}^{s_{-i}^{*} p}\right)$ does not hold.

Corollary 1 The system $\left(\mathbf{V}_{i}\right)$ holds if and only if either

$$
\bigvee_{\substack{k=1 \\ k \neq i}}^{N} \bigvee_{\substack{j=0 \\ l}}^{d_{k}} \bigvee_{\substack{l=1 \\ l>j}}^{d_{k}}\left(v_{k j}=0\right) \wedge\left(v_{k l}=0\right),
$$

or for some pure strategies $s_{k l} \in S_{k}$ for each $k \in I-\{i\}$ defining a $(-i)$-strategy profile $s_{-i}^{*} \in S_{-i}$, we have

$$
\begin{gathered}
\left(v_{i 0}, \ldots, v_{i d_{i}}\right)=q\left(s_{-i}^{*}\right), \\
v_{k l_{k}}=0 \quad \text { for each } k \in I-\{i\},
\end{gathered}
$$

and

$$
v_{k m_{k}} \neq 0 \text { for all } m_{k} \neq l_{k} \text {, for each } k \in I-\{i\} \text {. }
$$

Proof This follows from our characterization of $\left(\mathbf{V}_{i}\right)$, Lemmas 1 and 2. We impose the condition

$$
v_{k m_{k}} \neq 0 \text { for all } m_{k} \neq l_{k} \text {, for each } k \in I-\{i\}
$$

in the latter alternative because otherwise, the former alternative holds.

Suppose we have a solution of the system $(\mathbf{V})$ in which the latter alternative holds for some $i \in I$. As noted earlier, for a generic game, exactly one $v_{i j}=0$ (the one for which $u_{i}\left(s_{i j}, s_{-i}\right)-u_{i}\left(s_{i 0}, s_{-i}\right)$ attains its maximum, i.e., the one for which $u_{i}\left(s_{i j}, s_{-i}\right)$ is maximum) and the rest are zero. So the former alternative cannot hold for any $k \in I$. Define $l_{i}$ by $v_{i l_{i}}=0$, and let $s^{*}=\left(s_{i} l_{i}, s_{-i}^{*}\right) \in S$. For each $k \in I$, the payoff $u_{k}\left(s_{k l_{k}}, s_{-k}^{*}\right)$ must be the maximum among $u_{k}\left(s_{k l}, s_{-k}^{*}\right)$. Since $v_{k l} \neq 0$ for $l \neq l_{k}$, we must have $\sigma_{k l}=0$ for $l \neq l_{k}$, so $\sigma_{k l_{k}}=1$. That is, $\sigma$ is the pure strategy profile $s^{*}$. So this is the case of a pure strict Nash equilibrium $s^{*}$, that is, one for which the pure strategy $s_{k}^{*}$ is a strictly better response to $s_{-k}^{*}$ than any other pure strategy of $k$, for each $k \in I$.

Note well that this is a weaker condition than that $s^{*}$ be an equilibrium in dominant strategies. For example, consider a game of two players who each can take one of two actions, in which the payoff to each player is the same if they take the same action and strictly less if they take opposite actions. This game has two pure strict Nash equilibria, corresponding to both players taking the same one of the two actions. But neither of the actions is a dominated strategy for either of the players.

Suppose the latter alternative holds for some $i \in I$ and both $v_{i j}=0$ and $v_{i j^{\prime}}=0$ for some $j \neq j^{\prime}$. Then this makes the former alternative true for every $k \in I-\{i\}$. 
Proposition 2 Suppose $\sigma$ is a Nash equilibrium of a generic game, and $\sigma$ is not a pure strict Nash equilibrium. Then there are two players $i, k \in I$ with $i \neq k$ and two pure strategies each, $s_{i j_{0}}, s_{i} j_{1} \in S_{i}$ with $j_{0} \neq j_{1}$ and $s_{k l_{0}}, s_{k l_{k}} \in S_{k}$ with $l_{0} \neq l_{1}$, such that $u_{i}\left(s_{i j_{0}}, \sigma_{-i}\right)=u_{i}\left(s_{i j_{1}}, \sigma_{-i}\right)=u_{i}(\sigma)$ and $u_{k}\left(s_{k l_{0}}, \sigma_{-k}\right)=u_{k}\left(s_{k l_{1}}, \sigma_{-k}\right)=u_{k}(\sigma)$.

Proof The first alternative in Corollary 1 must hold for each $i \in I$. Pick a player $n \in I$. The condition

$$
\bigvee_{\substack{k=1 \\ k \neq n}}^{N} \bigvee_{j=0}^{d_{k}} \bigvee_{\substack{l=1 \\ l>j}}^{d_{k}}\left(v_{k j}=0\right) \wedge\left(v_{k l}=0\right)
$$

means there is $k \neq n$ and pure strategies $s_{k l_{0}}, s_{k l_{1}} \in S_{k}$ with $v_{k l_{0}}=v_{k l_{1}}=0$, i.e., $u_{k}\left(s_{k l_{0}}, \sigma_{-k}\right)=u_{k}\left(s_{k l_{1}}, \sigma_{-k}\right)=u_{k}(\sigma)$. This makes $\left(\mathbf{V}_{m}\right)$ hold for every player $m \in$ $I-\{k\}$. For $k$ itself, the condition $\bigvee_{\substack{i=1 \\ i \neq k}}^{N} \bigvee_{j=0}^{d_{i}} \bigvee_{\substack{l=1 \\ l>j}}^{d_{i}}\left(v_{i j}=0\right) \wedge\left(v_{i l}=0\right)$ means there is $i \neq k$ and pure strategies $s_{i} j_{0}, s_{i j_{1}} \in S_{i}$ with $u_{i}\left(s_{i j 0}, \sigma_{-i}\right)=u_{i}\left(s_{i j 1}, \sigma_{-i}\right)=u_{i}(\sigma)$.

In this case we cannot isolate $\sigma_{i} j_{0}$ and $\sigma_{i j_{1}}$ simply by looking at the best responses for $i$, and similarly with $k$. We must solve the polynomial system $(*)$.

As noted before, at least one $v_{n m}$ must vanish for every $n \in I$. In the conditions of the proposition, the least complex case is that exactly one $v_{n m}$ vanishes for each $n \in I-\{i, k\}$, say $v_{n m_{n}}$; that $v_{i j}>0$ for $j \neq j_{0}$ and $j \neq j_{1}$; and that $v_{k l}>0$ for $k \neq k_{0}$ and $k \neq k_{1}$. Then each player $n \in I-\{i, k\}$ executes pure strategy $s_{n m_{k}}$. Furthermore $i$ does not execute pure strategy $s_{i j}$ with any probability for $j \neq j_{0}$ and $j \neq j_{1}$, and $k$ does not execute pure strategy $s_{k l}$ with any probability for $l \neq l_{0}$ and $l \neq l_{1}$. Then the system $(*)$ reduces to the system for a game with two players, which we renumber as 1 and 2 , with two pure strategies each, which we renumber as $s_{10}$, $s_{11}, s_{20}$, and $s_{21}$. Write $u_{j l}^{i}=u_{i}\left(s_{1 j}, s_{2 l}\right)$. In this case the system $(*)$ is:

$$
\begin{gathered}
u_{00}^{1} \sigma_{20}+u_{01}^{1} \sigma_{21}=u_{10}^{1} \sigma_{20}+u_{11}^{1} \sigma_{21}, \\
u_{00}^{2} \sigma_{10}+u_{10}^{2} \sigma_{11}=u_{01}^{2} \sigma_{10}+u_{11}^{2} \sigma_{11}, \\
\sigma_{10}+\sigma_{11}=1, \\
\sigma_{20}+\sigma_{21}=1 .
\end{gathered}
$$

Substituting $1-\sigma_{11}$ for $\sigma_{10}$ and $1-\sigma_{21}$ for $\sigma_{20}$, we obtain

$$
\begin{aligned}
& \left(u_{11}^{1}-u_{10}^{1}-u_{01}^{1}+u_{00}^{1}\right) \sigma_{21}=u_{00}^{1}-u_{10}^{1}, \\
& \left(u_{11}^{2}-u_{10}^{2}-u_{01}^{2}+u_{00}^{2}\right) \sigma_{11}=u_{00}^{2}-u_{01}^{2}
\end{aligned}
$$

Notice that the equilibrium found by this system need not be totally mixed; for instance, $u_{00}^{1}-u_{10}^{1}$ could equal zero, in which case $\sigma_{2}=s_{20}$, or $u_{11}^{1}-u_{01}^{1}$ could equal zero. in which case $\sigma_{2}=s_{21}$. (If they are both zero, then player 1 has no control over player 1 's own payoff and hence every mixed strategy of player 1 is a best response. So the 
requirement that 1 play a best response, which leads to the first equation, does not impose any constraint on the strategy $\sigma_{21}$ of player 2.) Similarly, $u_{00}^{2}-u_{01}^{2}$ could equal zero, in which case $\sigma_{1}=s_{10}$, or $u_{11}^{2}-u_{10}^{2}$ could equal zero, in which case $\sigma_{1}=s_{11}$. Both of these cases may even occur, in which case the root of the system is a pure Nash equilibrium.

Open Access This article is distributed under the terms of the Creative Commons Attribution Noncommercial License which permits any noncommercial use, distribution, and reproduction in any medium, provided the original author(s) and source are credited.

\section{References}

Basu, S., Pollack, R., Roy, M.-F.: Algorithms in Real Algebraic Geometry. Heidelberg: Springer (2003) Bernstein, D.N.: The number of roots of a system of equations. Funct Anal Appl 9, 183-185 (1975)

Bochnak, J., Coste, M., Roy, M.-F.: Real Algebraic Geometry. Heidelberg: Springer (1998)

Cox, D., Little, J., O’Shea, D.: Ideals, Varieties, and Algorithms. Heidelberg: Springer (1997)

Cox, D., Little, J., O’Shea, D.: Using Algebraic Geometry. Heidelberg: Springer (1998)

Datta, R.S.: Algebraic Methods In Game Theory. Ph.D. thesis, University of California at Berkeley (2003a)

Datta, R.S.: Universality of Nash equilibria. Math Oper Res 28, 424-432 (2003b)

Datta, R.S.: Using computer algebra to find Nash equilibria. In: Proceedings of the 2003 International Symposium on Symbolic and Algebraic Computation, pp. 74-79 (electronic). ACM, New York (2003c)

Dickenstein, A., Emiris, I.Z. (eds.): Solving Polynomial Equations. Heidelberg: Springer (2005)

Gelfand, I.M., Kapranov, M.M., Zelevinsky, A.V.: Discriminants, Resultants and Multidimensional Determinants. Basel: Birkhäuser (1994)

Greuel, G.-M., Pfister, G., Schönemann, H.: Singular 2.0. A computer algebra system for polynomial computations. Centre for Computer Algebra, University of Kaiserslautern. http://www.singular.uni-kl.de (2001)

Harsanyi, J.: Oddness of the number of equilibrium points: a new proof. Int J Game Theory 2, 235-250 (1973)

Herings, P.J.-J., Peeters, R.: A globally convergent algorithm to compute all Nash equilibria for $n$-person games. Ann Oper Res 137, 349-368 (2005)

Herings, P.J.-J., Peeters, R.: Homotopy methods to compute equilibria in game theory. Econ Theory (2009) (this issue)

Huber, B., Sturmfels, B.: A polyhedral method for solving sparse polynomial systems. Math Comput 64, 1541-1555 (1995)

Kouchnirenko, A.G.: Newton polytopes and the Bezout theorem. Funct Anal Appl 10, 233-235 (1976)

Lazard, D., Rouillier, F.: Solving parametric polynomial systems. J Symb Comput 42, 636-667 (2007)

McKelvey, R., McLennan, A.: The maximal number of regular totally mixed Nash equilibria. J Econ Theory 72, 411-425 (1997)

McKelvey, R.D., McLennan, A.M., Turocy, T.L.: Gambit: Software tools for game theory, version 0.2006.01.20 (2006). Available at http://econweb.tamu.edu/gambit/

McLennan, A.M.: The expected number of real roots of a multihomogeneous system of polynomial equations. Am J Math 124, 49-73 (2002)

Montes, A.: A new algorithm for discussing Groebner bases with parameters. J Symb Comput 33, 183208 (2002)

Osborne, M.J., Rubinstein, A.: A Course in Game Theory. Cambridge: MIT Press (1994)

Porter, R., Nudelman, E., Shoham, Y.: Simple search methods for finding a Nash equilibrium. Games Econ Behav 63, 642-662 (2008)

Sommese, A.J., Wampler, C.W.: The Numerical Solution of Systems of Polynomials Arising in Engineering and Science. Singapore: World Scientific (2005)

Sturmfels, B.: Solving Systems of Polynomial Equations. American Mathematical Society, Providence (2002)

Torregrosa, J.R., Jordán, C., el Ghamry, R.: The nonsingular matrix completion problem. Int J Contemp Math Sci 2, 349-355 (2007)

Verschelde, J.: Algorithm 795: PHCpack: a general-purpose solver for polynomial systems by homotopy continuation. ACM Trans Math Softw 25, 251-276 (1999) 